\title{
The analysis, identification and measures to remove inconsistencies from differential evolution mutation variants
}

\author{
Qamar Abbas $^{\mathrm{a}, *}$, Jamil Ahmad ${ }^{\mathrm{b}}$, Hajira Jabeen ${ }^{\mathrm{c}}$ \\ a Department of Computer Science and Software Engineering, International Islamic University, \\ Islamabad, 44000, Pakistan \\ b Vice Chancellor, Kohat University of Science and Technology (KUST), Kohat, 44000, Pakistan \\ c Computer Department, Iqra University, Islamabad, 44000, Pakistan
}

*Corresponding author, e-mail: qamar.abbas@iiu.edu.pk

Received 1 Dec 2014

Accepted 1 Jun 2017

\begin{abstract}
Differential evolution (DE) is a powerful global optimization algorithm which has been studied intensively by many researchers in recent years. A number of mutation variants have been established for this algorithm. These mutation variants make the $\mathrm{DE}$ algorithm more applicable, but random development of these variants has created inconsistencies such as naming and formulation. Hence this study aims to identify inconsistencies and to propose solutions to make them consistent. Most of the inconsistencies exist because of the uncommon nomenclature used for these variants. In this study, a comprehensive study is carried out to identify inconsistencies in the nomenclature of mutation variants that do not match each other. Appropriate and consistent names are proposed for them. The proposed names assigned to conflicting variants are based on the name of the variant, the total number of vectors used to generate the trial vector, and the order of the vectors to form the equation of these mutation variants. To ensure the performance diversity of the consistent set of DE mutation strategies, experimental results are generated using a test suit of benchmark functions.
\end{abstract}

KEYWORDS: crossover, optimization, algorithm

\section{INTRODUCTION}

Differential evolution (DE), proposed by Storn and Price $^{1}$, is a stochastic-population-based evolutionary algorithm. DE is simple, easy to use, and speedy, and has a greater probability to find the global optima for any given function ${ }^{2,3}$. DE has been successfully used in various systems such as electrical power systems ${ }^{4}$, microwave engineering ${ }^{5}$, robotics $^{6}$, bioinformatics ${ }^{7}$, chemical engineering ${ }^{8}$, pattern recognition ${ }^{9}$, artificial neural networks ${ }^{10}$, and signal processing ${ }^{11}$. In DE algorithm, a population of potential solutions is randomly initialized within an $n$-dimensional search space. All potential solutions are equally likely to be selected as a parent in DE algorithm. The candidate solutions evolve themselves by exploring the entire search space over time to locate the optima of the objective function. A new vector is generated by adding the weighted difference between two population vectors to a third vector at each iteration of DE algorithm. Three vectors, randomly selected from the existing population, are used to generate each new vector. Many algorithms are used for numerical benchmark optimization, but DE has shown better performance than genetic algorithms and particle swarm optimization for such problems ${ }^{1,12}$. There are many parameters in DE algorithm such as the population size $N_{\mathrm{p}}$, mutation probability $F$, and crossover $C_{r}$. DE algorithm has many mutation strategies ${ }^{2}$ in the literature. Various state-of-the-art versions of DE algorithms such as $\mathrm{ADE}^{13}, \mathrm{jDE}^{2}, \mathrm{SaDE}^{14}, \mathrm{JADE}^{15}, \mathrm{DEGL}^{16}, \mathrm{CoDE}^{17}$, $\mathrm{EPSDE}^{18}, \mathrm{MDE}_{\mathrm{p}} \mathrm{BX}^{19}$, and $\mathrm{FADE}^{20}$ are based on parameter selection, parameter adaption, strategy selection, and/or strategy adaption mechanisms. Several adaptive and self-adaptive mechanisms in DE are used for parameter selection/adaption and various strategies for pool selection/adaption. DE state-of-the-art proved to be very powerful by using conventional DE mutation variants to form a strategy pool in strategy selection/adaption or using parameter selection/adaption along with some conventional trial vector generation scheme. Some 
parameter and strategy adaption/selection schemes of DE state-of-the-art variant related work are discussed in this section.

Zaharie $^{13}$ introduced a parameter adaption scheme in his research work to control the diversity in the population. He used the concept of multipopulation for the diversity in the population that can help to avoid premature convergence. The experimental result showed promising performance for the proposed approach. Liu and Lampinen ${ }^{20}$ introduced fuzzy-based control parameter adaption (FADE) in their research. They used a fuzzy controller to adopt the values of control parameters $F$ and $C_{r}$ in successive generations for the crossover and mutation operations of $\mathrm{DE}$ algorithm. Brest et $\mathrm{al}^{2}$ introduced an adaptive control parameter setting of DE (jDE) by encoding $F$ and $C_{r}$ into individuals. They introduced $\tau_{1}$ and $\tau_{2}$ to control $F$ and $C_{r}$ parameter values in DE algorithm. The values of $\tau_{1}=0.1, \tau_{2}=0.1, F_{u}=0.9, F_{l}=0.1$ were used in their proposed adaption mechanism. In this technique, better individuals have opportunity to survive by propagating their better parameter values to produce new offspring. Qin et $\mathrm{al}^{14}$ introduced both control parameter adaption as well as a strategy adaption mechanism in DE algorithm (SaDE). In the strategy adaption scheme, they used a strategy candidate pool of four strategies: $\mathrm{DE} / \mathrm{rand} / 1, \mathrm{DE} / \mathrm{rand} / 2, \mathrm{DE} / \mathrm{rand}$ to best/2, and $\mathrm{DE} /$ current to rand/1. Each target vector generates a trial vector based on the learning period over previous generations that is based on the success rate of previous generations in the experiment. In parameter adaption, $\mathrm{SaDE}$ adjusts the control parameter $C_{r}$ based on the median value of $C_{r}$ that is calculated based on previous $C_{r}$ values that have successfully generated a trial vector. Zhang and Sanderson ${ }^{15}$ introduced a new parameter adaption method and used the DE/current to pbest/1 strategy in their research work. In the parameter adaption, at each generation, $C_{r}$ is generated by a normal distribution and $F$ is generated by a Cauchy distribution to smoothly update these parameters. The DE/current to pbest/1 strategy randomly selects the best population member from the top $100 \%$ population members of the current population. They used the concept that incorporation of a random best individual can enhance the searching capability of DE algorithm. JADE uses the concept of external archive to store the recently explored inferior solution and then select the new population members from the union of the current population and the external archive so that less performing individuals have a chance to be part of the population. Das et $\mathrm{al}^{16}$ used $\mathrm{DE} /$ target to best/1 by utilizing the concept of neighbourhood of each population individual in order to balance the exploration and exploitation abilities of DE algorithm. They introduced local- and global-neighbourhood DE (NSDE/DEGL) schemes and combined both local and global neighbourhood schemes to generate the donor vector. In NSDE, $\alpha$ and $\beta$ are scaling factors, a local donor vector is created by employing the best vector in the neighbourhood and two other random vectors but the global best uses the entire population best vector and other two any random vectors from the current population. In DEGL, exploration and exploitation are controlled by a weight factor $\omega$. They discussed increasing the weight factor, random weight factor, and self adaptive weight factor in their research work. Mallipeddi et al $^{18}$ introduced an ensemble-based crossover and mutation DE strategy (EPSDE) and their corresponding control parameter scheme in their research work. They used a pool of different crossover and mutation strategies and a pool of values for each associated control parameter. Each target vector generates a trial vector based on the assigned strategy and the parameter values. Successful combinations of the mutation and crossover strategy and associated parameter values are stored in the pool. EPSDE uses DE/current to rand/1 and JADE mutation strategy along with binomial and exponential crossover. Minhazul Islam et $\mathrm{al}^{19}$ proposed a new mutation strategy, a modification in the conventional binomial crossover scheme, and a parameter adaption scheme in DE algorithm. They used $\mathrm{DE} /$ current to gr_best/ 1 and called it a less greedy version of $\mathrm{DE} /$ current to best/1. The proposed mutation strategy $\mathrm{DE} /$ current to gr_best/1 uses the best vector from $q \%$ population of individuals to generate the trial vector for each target vector. In pbest, a crossover mutant vector can swap the $p$-top ranked individuals of the current generation instead of current parent using a binomial crossover scheme. In parameter adaption scheme, scale factor adaption is based on the Cauchy, and crossover probability adaption is based on the Gaussian distribution. Wang, Cai, and Zhang ${ }^{17}$ introduced the composite DE (CoDE) variant. In this scheme, they used a pool of three trial vector generation strategies and a pool of three parameter setting combinations. The trial vector strategies used are rand/1, rand/2, and current to rand/1, while the parameter setting combinations are $\left[F=1.0, C_{r}=0.1\right],\left[F=1.0, C_{r}=\right.$ 
0.9], and $\left[F=0.8, C_{r}=0.2\right]$. To generate a new solution in CoDE, each strategy is coupled with a randomly chosen parameter setting. Gong et $\mathrm{al}^{21}$ introduced a new strategy adaptation mechanism (SaM) in their research work. They combined SaM with JADE and named it SaJADE. A novel strategy parameter $\eta_{i} \in[0,1)$ was used to control the selection of any strategy from the strategy pool. They chose four various DE strategies DE/current to pbest without archive, DE/rand to pbest without archive, DE/current to pbest with archive, and $\mathrm{DE} / \mathrm{rand}$ to pbest with archive to form a strategy pool.

The popularity of DE due to its advantages over other evolutionary methods can be observed in its diverse applications in real life fields ${ }^{22-29}$. But the intensive and random development of DE algorithm has created several inconsistencies in the naming and formulation of trial vector generation schemes. The inconsistencies in DE algorithm may reduce the attraction of new researchers to use this algorithm in problem solving. It is important to mention that this study does not mean to prove any weakness in $\mathrm{DE}$ algorithm or criticize the effort of any researcher, but to make this algorithm easier and categorically clearer. Probably due to inconsistencies in DE mutation variants, there are some powerful conventional $\mathrm{DE}$ variants that are not announced to be powerful variants in the literature, while having either dominating or comparable performance as compared to those variants which are commonly used or used in DE state-of-the-art. Focusing on this, an effort is made in this study to present a consistent set of variants that $\mathrm{DE}$ has a number of variants and only few are commonly used that may also be due to inconsistency in most of DE variants. This study may not be a complete effort in this direction, but this attempt will prove to be significant addition in $\mathrm{DE}$ literature.

\section{DE ALGORITHM}

DE algorithm has three different parameters: a population of size $N_{\mathrm{p}}$, a crossover control parameter $C_{r}$, and a difference vector amplification parameter $F$. Each population member in $\mathrm{DE}$ is represented as a $D$-dimensional parameter vector. In $\mathrm{DE}$ algorithm, the population is initialized randomly and is supposed to cover the entire search space. Each vector in the DE is represented by $\mathbf{x}_{i, g}$, where $i=$ $1,2,3, \ldots, N_{\mathrm{p}}$ and $g$ is generation number. New offsprings in DE algorithm are generated by mutation, crossover, and selection operators. The repair operator proposed by Wang ${ }^{30}$ is also used in this study. Three different terminologies of vectors: donor vector, trial vector, and target vector are used in DE algorithm. Donor vector is a vector that is created in the mutation operation, trial vector is created in the crossover operation, and target vector is the current vector of population.

Mutation: In the mutation operation, a mutant vector, also called donor vector, is created. The donor vector $\mathbf{v}_{i, g}$ of the $i$ th population member is calculated by adding the weighted difference of two vectors to the third vector:

$$
\mathbf{v}_{i, g}=\mathbf{x}_{r_{1}, g}+F\left(\mathbf{x}_{r_{2}, g}-x_{\mathbf{r}_{3}, g}\right),
$$

where indices $r_{1}, r_{2}, r_{3} \in\left\{1,2,3, \ldots, N_{\mathrm{p}}\right\}$ are randomly selected to be different from $i$ and $F$ is the mutation probability parameter.

Crossover: DE crossover strategies control the number of inherited components from the mutant vector to form a target vector. Binomial and exponential are main crossover schemes ${ }^{16,31}$. The DE crossover rate parameter $C_{r}$ influences the size of the perturbation of the base (target) vector to ensure the population diversity ${ }^{17}$.

Binomial crossover: In the crossover operation of $\mathrm{DE}$ algorithm, a trial vector is formed. In the binomial crossover scheme, the trail vector $\mathbf{u}_{i, g}=$ $\left\langle u_{i, 1, g}, u_{i, 2, g}, \ldots, u_{i, D, g}\right\rangle$ is generated by the equation, for $i=1,2, \ldots, N_{\mathrm{p}}, j=1,2, \ldots, D$ :

$$
u_{i, j, g}= \begin{cases}v_{i, j, g} & \left(\operatorname{rand}_{j} \leqslant C_{r} \text { or } j=j_{\mathrm{rd}}\right) \\ x_{i, j, g}, & \text { otherwise, }\end{cases}
$$

where $j_{\mathrm{rd}}$ is a randomly chosen integer in the range $[1, D]$, rand $_{j}$ is a random number in $(0,1), \mathbf{v}_{i, g}$ is the donor vector, and $C_{r} \in(0,1)$ is the crossover control parameter. Due to the range of $j_{\text {rand }}, \mathbf{u}_{i, g}$ is always different from $\mathbf{x}_{i, g}$.

Exponential crossover: In the exponential crossover scheme, the trail vector $\mathbf{u}_{i, g}=$ $\left\langle u_{i, 1, g}, u_{i, 2, g}, \ldots, u_{i, D, g}\right\rangle$ is created as:

$$
u_{i, j, g}=\left\{\begin{array}{cc}
v_{i, j, g} & \text { if } j \in\left\{l,\langle l+1\rangle_{D}, \ldots,\langle l+L-1\rangle_{D}\right\} \\
\quad \text { and }\left(\operatorname{rand}_{j} \leqslant C_{r}\right), \\
x_{i, j, g} \quad \text { otherwise, }
\end{array}\right.
$$

for $i=1,2, \ldots, N_{\mathrm{p}}, j=1,2,3, \ldots, D$, where $\langle\cdot\rangle_{D}$ denotes the modulo function with modulus $D$. The starting index $l$ is chosen at random from $[1, D]$ and $L$ is also a randomly generated number from $[1, D]$. The parameters $l$ and $L$ are regenerated for each trial vector $\mathbf{u}_{i, g}$.

Selection: In DE algorithm, new population members are formed using the selection operation. 
The selection operator uses the greedy approach by comparing the fitness of trial vector $\mathbf{u}_{i, g}$ with the fitness of target $\mathbf{x}_{i, g}$; the vector having best fitness is selected as a member of the new population:

$$
\mathbf{x}_{i+1, g}= \begin{cases}\mathbf{u}_{i, g} & \text { fitness }\left(\mathbf{u}_{i, g}\right)<\text { fitness }\left(\mathbf{x}_{i, g}\right) \\ \mathbf{x}_{i, g}, & \text { otherwise }\end{cases}
$$

where the fitness function calculates the fitness value of the objective function.

\section{DE MUTATION VARIANTS, LITERATURE INCONSISTENCY AND SUGGESTED CORRECTIONS}

There are several DE algorithm mutation variants/strategies that are formed by the linear combination of existing population members. The trial vector and target vector form the mutant vector in $\mathrm{DE}$. Throughout this paper, $x_{i}$ denotes the current target vector, $i$ is the running index, $u_{i}$ represents the trial vector, and $v_{i}$ is a mutant vector. In DE algorithm, different mutation schemes are used to create the trial vector by using any combination of current, best, and random vectors. The behaviour of $\mathrm{DE}$ algorithm is influenced by the selection of mutation strategy and crossover scheme along with their control parameters: mutation probability $F$ and crossover rate ${ }^{31,32} C_{r}$. The difference vector is the difference of two mutating vectors that is used to form offspring in the population ${ }^{33}$. To form the mutant vector in DE, some researchers use a random value $\lambda \in(0,1)$ as a coefficient multiplier with the first difference vector ${ }^{1,34}$ and mutation probability $F$ as a coefficient multiplier with the other difference vectors $^{34,35}$. Some researchers have used only $F$ as a coefficient multiplier with the difference vectors to form the mutant vector ${ }^{31,36}$. To reduce the number of control parameters of DE algorithm, we use ${ }^{37} \lambda=$ $F$. This section contains the detail of DE mutation variants that reveals several irregularities in naming and formulation of DE mutation strategies. Before describing in detail the DE mutation variants, it is important to understand the vectors associated with DE mutation strategies. DE mutation strategies can be formed by the combinations of current, random, better, and best vectors. In any mutation strategy, the order, number, and name of vectors are very important. Throughout the analysis, $x_{g}^{k}$ denotes the $k$ th random vector for $g$ th generation, $v_{g}^{i}$ is the $i$ th component of donor vector at $g$ th generation, $x_{g}^{\text {best }}$ states the best vector at $g$ th generation, $x_{g}^{i}$ denotes the current vector, also called target vector, at $g$ th generation, and $x_{g}^{\text {better }}$ states the better vector at $g$ th generation.

The mutation strategies discussed below contain several inconsistencies that are identified in the next sections. The trial vector generation of each variant is presented by using selected population members that are based on the equations of associated variants. The important aspect is the order of vectors in the equation of variant and total number of vectors used to generate the trial vector generation for any variant.

$\left(\mathrm{v}_{1}\right) \mathrm{DE} / \mathrm{rand} / 1$ was introduced by Storn \& Price ${ }^{1}$. This strategy is known as the basic strategy in the DE algorithm. DE/rand/1 is used as a default variant in the standard $\mathrm{DE}$ algorithm. This mutation strategy has no conflicts in the literature with other mutation strategies. The equation is

$$
v_{g}^{i}=x_{g}^{r_{1}}+F\left(x_{g}^{r_{2}}-x_{g}^{r_{3}}\right),
$$

which uses three random vectors $x_{g}^{r_{1}}, x_{g}^{r_{2}}, x_{g}^{r_{3}}$ and one difference vector to generate the mutant vector. This strategy perturbs a based random vector $x_{g}^{r_{1}}$ with one weighted difference vector of random vectors.

$\left(v_{2}\right)$ DE/best/1 was introduced by Storn ${ }^{34}$ for function optimization application. This mutation strategy has no conflicts with other mutation strategies in the literature. The equation is

$$
v_{g}^{i}=x_{g}^{\text {best }}+F\left(x_{g}^{r_{2}}-x_{g}^{r_{3}}\right),
$$

which contains two random vectors $x_{g}^{r_{1}}, x_{g}^{r_{2}}$ in the difference vector and one best vector $x_{g}^{\text {best }}$ to generate the mutant vector. This variant perturbs the best vector $x_{g}^{\text {best }}$ with a weighted difference vector of random vectors.

$\left(v_{3}\right) \mathrm{DE} / \mathrm{rand} / 2$ was introduced by Storn \& Price ${ }^{31}$ for function optimization application. This mutation strategy has no conflicts with other mutation strategies in the literature. The equation is

$$
v_{g}^{i}=x_{g}^{r_{1}}+F\left(x_{g}^{r_{2}}-x_{g}^{r_{3}}\right)+F\left(x_{g}^{r_{4}}-x_{g}^{r_{5}}\right),
$$

which uses five random vectors $x_{g}^{r_{1}}, x_{g}^{r_{2}}, x_{g}^{r_{3}}, x_{g}^{r_{4}}$, $x_{g}^{r_{5}}$ in perturbation of a based random vector $x_{g}^{r_{1}}$ using two weighted difference vectors to generate the mutant vector. This results in a new vector that is not biased towards a particular direction.

$\left(\mathrm{v}_{4}\right) \mathrm{DE} /$ best $/ 2$ mutation strategy was introduced by Price ${ }^{37}$ for function optimization problems. 
This mutation strategy has no conflicts with other mutation strategies in the literature. The equation is

$$
v_{g}^{i}=x_{g}^{\text {best }}+F\left(x_{g}^{r_{1}}-x_{g}^{r_{2}}\right)+F\left(x_{g}^{r_{3}}-x_{g}^{r_{4}}\right),
$$

which perturbs $x_{g}^{\text {best }}$ using four random vectors $x_{g}^{r_{1}}, x_{g}^{r_{2}}, x_{g}^{r_{3}}, x_{g}^{r_{4}}$ in two weighted difference vectors. This variant consumes best vector along with two weighted difference vectors without repeating any vector.

$\left(v_{5}\right)$ DE/current to rand/1 mutation strategy was used by many researchers ${ }^{38-40}$. This mutation strategy has naming conflict with other mutation strategies in the literature. The equation is

$$
v_{g}^{i}=x_{g}^{i}+F\left(x_{g}^{r_{1}}-x_{g}^{i}\right)+F\left(x_{g}^{r_{2}}-x_{g}^{r_{3}}\right),
$$

which places the perturbation at a location between the current population member $x_{\sigma}^{i}$ and a randomly chosen population member $x_{g}^{g}$ and uses a weighted difference vector of random vectors. This scheme uses three random vectors $x_{g}^{r_{1}}, x_{g}^{r_{2}}, x_{g}^{r_{3}}$ and the current vector $x_{g}^{i}$ to generate the mutant vector.

$\left(\mathrm{v}_{6}\right) \mathrm{DE} /$ current to rand/1 mutation strategy was used by Qin et $\mathrm{al}^{14}$ for constrained real parameter optimization. This mutation strategy has a naming conflict with other mutation strategies. The equation is

$$
v_{g}^{i}=x_{g}^{r_{1}}+F\left(x_{g}^{r_{2}}-x_{g}^{i}\right)+F\left(x_{g}^{r_{1}}-x_{g}^{r_{3}}\right),
$$

which places the perturbation at a location away from a random vector $x_{g}^{r_{1}}$ between the current vector and another random vector. This scheme contains three random vectors $x_{g}^{r_{1}}, x_{g}^{r_{2}}, x_{g}^{r_{3}}$ and the current vector $x_{g}^{i}$ in the perturbation by repeating a random number $x_{g}^{r_{1}}$ in the weighted difference vector.

$\left(\mathrm{v}_{7}\right) \mathrm{DE} /$ current to best/1 mutation strategy, introduced by Storn \& Price ${ }^{1}$, was used by many researchers ${ }^{41,42}$. This mutation strategy has a naming and equation conflict with other mutation strategies in literature. The equation is

$$
v_{g}^{i}=x_{g}^{i}+F\left(x_{g}^{\text {best }}-x_{g}^{i}\right)+F\left(x_{g}^{r_{1}}-x_{g}^{r_{2}}\right),
$$

which places the perturbation at a location between the current vector $x_{g}^{i}$ and the best vector $x_{g}^{\text {best }}$ at current generation. The variant use a weighted difference of random vectors $x_{g}^{r_{1}}, x_{g}^{r_{2}}$ in perturbing the current vector $x_{g}^{i}$. $\left(\mathrm{v}_{8}\right) \mathrm{DE} /$ current to best/1 mutation strategy was used in Podoba et $\mathrm{al}^{43}$ for surface reconstruction using AI. This mutation strategy has a naming conflict as well as the equation conflict with other mutation strategies in the literature. The equation is

$$
v_{g}^{i}=x_{g}^{i}+F\left(x_{g}^{\text {best }}-x_{g}^{r_{1}}\right)+F\left(x_{g}^{r_{1}}-x_{g}^{r_{2}}\right),
$$

which perturbs the current vector $x_{g}^{i}$ by moving the perturbation at a location between the best vector $x_{g}^{\text {best }}$ and a random vector $x_{g}^{r_{1}}$. The variant utilizes two random vectors $x_{g}^{r_{1}}, x_{g}^{r_{2}}$, the best vector $x_{g}^{\text {best }}$, and the current vector $x_{g}^{i}$ in perturbation with one weighted difference vector by repeating a random vector $x_{g}^{r_{1}}$.

$\left(\mathrm{v}_{9}\right) \mathrm{DE} / \mathrm{rand}$ to best/1 mutation strategy was used in Davendra et $\mathrm{al}^{44}$ for travelling salesman problem. This mutation strategy has a naming and equation conflict with the other mutation strategies in the literature. The equation is

$$
v_{g}^{i}=x_{g}^{i}+F\left(x_{g}^{\text {best }}-x_{g}^{r_{1}}\right)+F\left(x_{g}^{r_{1}}-x_{g}^{r_{2}}\right),
$$

which perturbs the current vector $x_{g}^{i}$ by moving the at perturbation location between the best vector $x_{g}^{\text {best }}$ and a random vector $x_{g}^{r_{1}}$. This perturbation uses two random vectors $x_{g}^{r_{1}}, x_{g}^{r_{2}}$, the best vector $x_{g}^{\text {best }}$, and the current vector $x_{g}^{l}$ with one weighted difference vector by repeating a random vector $x_{g}^{r_{1}}$.

$\left(\mathrm{v}_{10}\right) \mathrm{DE} / \mathrm{rand}$ to best/1 mutation strategy was introduced by Storn ${ }^{34}$ for function optimization application. This mutation strategy has been used by many researchers ${ }^{18,45}$. This mutation strategy has naming conflict as well as equation conflict with other mutation strategies in the literature. The equation is

$$
v_{g}^{i}=x_{g}^{i}+F\left(x_{g}^{\text {best }}-x_{g}^{i}\right)+F\left(x_{g}^{r_{1}}-x_{g}^{r_{2}}\right),
$$

which perturbs the current vector $x_{g}^{i}$ by moving perturbation at location between the current vector $x_{g}^{i}$ and the best vector $x_{g}^{\text {best }}$. This perturbation use two random vectors $x_{g}^{r_{1}}, x_{g}^{r_{2}}$, the best vector $x_{g}^{\text {best }}$, and the current vector $x_{g}^{i}$ with one weighted difference vector.

$\left(\mathrm{v}_{11}\right) \mathrm{DE} / \mathrm{rand}$ to best/1 mutation strategy was used by many researchers ${ }^{46}$. This mutation strategy has naming conflict with other mutation strategies in literature. The equation is

$$
v_{g}^{i}=x_{g}^{r_{1}}+F\left(x_{g}^{\text {best }}-x_{g}^{r_{2}}\right)+F\left(x_{g}^{r_{3}}-x_{g}^{r_{4}}\right),
$$

which perturbs a random vector $x_{g}^{r_{1}}$ by moving the perturbation at location between the best 
vector $x_{g}^{\text {best }}$ and a random vector $x_{g}^{r_{2}}$. This perturbation uses four random vectors $x_{g}^{r_{1}}, x_{g}^{r_{2}}, x_{g}^{r_{3}}$, $x_{g}^{r_{4}}$ and the best vector $x_{g}^{\text {best }}$ with one weighted difference vector.

$\left(\mathrm{v}_{12}\right) \mathrm{DE} / \mathrm{rand}$ to best/1 mutation strategy was used by Almeida-Luz et $\mathrm{al}^{47}$, Mendes $\&$ Mohais ${ }^{48}$. This mutation strategy has naming conflict with other mutation strategies in the literature. The equation is

$$
v_{g}^{i}=x_{g}^{r_{1}}+F\left(x_{g}^{\text {best }}-x_{g}^{r_{1}}\right)+F\left(x_{g}^{r_{2}}-x_{g}^{r_{3}}\right),
$$

which perturbs a random vector $x_{g}^{r_{1}}$ by moving the perturbation at location between $x_{g}^{r_{1}}$ and the best vector $x_{g}^{\text {best }}$. This perturbation contains three random vectors $x_{g}^{r_{1}}, x_{g}^{r_{2}}, x_{g}^{r_{3}}$ and the best vector $x_{g}^{\text {best }}$ with a weighted difference vector.

$\left(\mathrm{v}_{13}\right) \mathrm{DE} / \mathrm{rand}$ to best/1 mutation strategy was used by Jeyakumar \& Velayutham ${ }^{40}$ for empirical analysis of DE variants over function optimization problem. This mutation strategy has naming conflict with other mutation strategies in the literature. The equation is

$$
v_{g}^{i}=x_{g}^{r_{1}}+F\left(x_{g}^{\text {best }}-x_{g}^{i}\right)+F\left(x_{g}^{r_{2}}-x_{g}^{r_{3}}\right),
$$

which perturbs a random vector $x_{g}^{r_{1}}$ by moving the perturbation at location between the best vector $x_{g}^{\text {best }}$ and the current vector $x_{g}^{i}$. This perturbation contains three random vectors $x_{g}^{r_{1}}$, $x_{g}^{r_{2}}, x_{g}^{r_{3}}$, the current vector $x_{g}^{i}$ and the best vector $x_{g}^{\text {best }}$ with one weighted difference vector.

$\left(\mathrm{v}_{14}\right) \mathrm{DE} /$ current to best/2 mutation strategy was used by many researchers ${ }^{49,50}$ for various problems. This mutation strategy has naming and equation conflicts with other mutation strategies in the literature. The equation is

$$
v_{g}^{i}=x_{g}^{i}+F\left(x_{g}^{\text {best }}-x_{g}^{i}\right)+F\left(x_{g}^{r_{1}}-x_{g}^{r_{2}}\right),
$$

which perturbs the current vector $x_{g}^{i}$ by moving the perturbation at location between the best vector $x_{g}^{\text {best }}$ and the current vector $x_{g}^{i}$. This perturbation contains two random vectors $x_{g}^{r_{1}}$, $x_{g}^{r_{2}}$, the best vector $x_{g}^{\text {best }}$ and the current vector $x_{g}^{i}$ with one weighted difference vector.

$\left(\mathrm{v}_{15}\right) \mathrm{DE} /$ current to best/2 mutation strategy was used by many researchers ${ }^{14,38,51}$. This mutation strategy has naming conflict with other mutation strategies in literature. The equation of is

$$
\begin{aligned}
v_{g}^{i}=x_{g}^{i} & +F\left(x_{g}^{\text {best }}-x_{g}^{i}\right) \\
& +F\left(x_{g}^{r_{1}}-x_{g}^{r_{2}}\right)+F\left(x_{g}^{r_{3}}-x_{g}^{r_{4}}\right),
\end{aligned}
$$

which perturbs the current vector $x_{g}^{i}$ by moving the perturbation at location between the best vector $x_{g}^{\text {best }}$ and the current vector $x_{g}^{i}$. This perturbation contains four random vectors $x_{g}^{r_{1}}$, $x_{g}^{r_{2}}, x_{g}^{r_{3}}$, the best vector $x_{g}^{\text {best }}$ and the current vector $x_{g}^{i}$ with two weighted difference vectors.

$\left(\mathrm{v}_{16}\right) \mathrm{DE} /$ current to rand/2 mutation strategy was used by Zielinski et $\mathrm{al}^{38}$ for choosing suitable variants of differential evolution in particle swarm optimization. This mutation strategy has naming conflict with other mutation strategies in the literature. The equation is

$$
\begin{aligned}
v_{g}^{i}=x_{g}^{i} & +F\left(x_{g}^{r_{1}}-x_{g}^{i}\right) \\
& +F\left(x_{g}^{r_{2}}-x_{g}^{r_{3}}\right)+F\left(x_{g}^{r_{4}}-x_{g}^{r_{5}}\right),
\end{aligned}
$$

which perturbs the current vector $x_{g}^{i}$ by moving the perturbation at location between a random vector $x_{g}^{r_{1}}$ and the current vector $x_{g}^{i}$. This perturbation contains five random vectors $x_{g}^{r_{1}}$, $x_{g}^{r_{2}}, x_{g}^{r_{3}}, x_{g}^{r_{4}}, x_{g}^{r_{5}}$ and the current vector $x_{g}^{i}$ with two weighted difference vectors.

$\left(\mathrm{v}_{17}\right) \mathrm{DE} / \mathrm{rand}$ to best/2 mutation strategy was used by Podoba et $\mathrm{al}^{43}$ for surface construction using AI. This mutation strategy has naming conflict with other mutation strategies in the literature. The equation is

$$
v_{g}^{i}=x_{g}^{r_{1}}+F\left(x_{g}^{\text {best }}-x_{g}^{r_{2}}\right)+F\left(x_{g}^{r_{3}}-x_{g}^{r_{4}}\right),
$$

which perturbs a random vector $x_{g}^{r_{1}}$ by moving its perturbation location towards location between the best vector $x_{g}^{\text {best }}$ and a random vector $x_{g}^{r_{2}}$. This perturbation contains four random vectors $x_{g}^{r_{1}}, x_{g}^{r_{2}}, x_{g}^{r_{3}}, x_{g}^{r_{4}}$ and the best vector $x_{g}^{\text {best }}$ with one weighted difference vector.

$\left(\mathrm{v}_{18}\right) \mathrm{DE} / \mathrm{rand}$ to best/2 mutation strategy was used by Oliveira \& Saramago ${ }^{52}$. This mutation strategy has naming and equation conflicts with the other mutation strategies in the literature. The equation is

$$
v_{g}^{i}=x_{g}^{i}+F\left(x_{g}^{\text {best }}-x_{g}^{i}\right)+F\left(x_{g}^{r_{1}}-x_{g}^{r_{2}}\right),
$$

which perturbs the current vector $x_{g}^{i}$ by moving the perturbation at location between the best vector $x_{g}^{\text {best }}$ and the current vector $x_{g}^{i}$. This perturbation contains two random vectors $x_{g}^{r_{1}}$, $x_{g}^{r_{2}}$, the best vector $x_{g}^{\text {best }}$ and the current vector $x_{g}^{l}$ with one weighted difference vector.

$\left(\mathrm{v}_{19}\right) \mathrm{DE} / \mathrm{rand}$ to best/2 mutation strategy was used by Weber ${ }^{53}$ on parallel global optimization. This mutation strategy has naming conflict 
with other mutation strategies in the literature. The equation is

$$
\begin{aligned}
v_{g}^{i}=x_{g}^{r_{1}} & +F\left(x_{g}^{\text {best }}-x_{g}^{i}\right) \\
& +F\left(x_{g}^{r_{2}}-x_{g}^{r_{3}}\right)+F\left(x_{g}^{r_{4}}-x_{g}^{r_{5}}\right),
\end{aligned}
$$

which perturbs a random vector $x_{g}^{r_{1}}$ by moving its perturbation location towards location between the best vector $x_{g}^{\text {best }}$ and the current vector $x_{g}^{i}$. This perturbation contains five random vectors $x_{g}^{r_{1}}, x_{g}^{r_{2}}, x_{g}^{r_{3}}, x_{g}^{r_{4}}, x_{g}^{r_{5}}$, the current vector $x_{g}^{i}$ and the best vector $x_{g}^{\text {best }}$ with two weighted difference vectors.

$\left(\mathrm{v}_{20}\right) \mathrm{DE} / \mathrm{rand}$ to best/2 mutation strategy was used by many researchers $45,54,55$. This mutation strategy has naming conflict with other mutation strategies in literature. The equation is

$$
\begin{aligned}
v_{g}^{i}=x_{g}^{i} & +F\left(x_{g}^{\text {best }}-x_{g}^{i}\right) \\
& +F\left(x_{g}^{r_{1}}-x_{g}^{r_{2}}\right)+F\left(x_{g}^{r_{3}}-x_{g}^{r_{4}}\right),
\end{aligned}
$$

which perturbs the current vector $x_{g}^{i}$ by moving the perturbation at location between the best vector $x_{g}^{\text {best }}$ and the current vector $x_{g}^{i}$. This perturbation contains four random vectors $x_{g}^{r_{1}}$, $x_{g}^{r_{2}}, x_{g}^{r_{3}}, x_{g}^{r_{4}}$, the current vector $x_{g}^{i}$ and the best vector $x_{g}^{\text {best }}$ with two weighted difference vectors.

$\left(\mathrm{v}_{21}\right) \mathrm{DE} / \mathrm{rand}$ to best/2 mutation strategy was used by many researchers $39,56,57$. This mutation strategy has naming conflict with other mutation strategies in literature. The equation is

$$
\begin{aligned}
v_{g}^{i}=x_{g}^{r_{1}} & +F\left(x_{g}^{\text {best }}-x_{g}^{r_{1}}\right) \\
& +F\left(x_{g}^{r_{2}}-x_{g}^{r_{3}}\right)+F\left(x_{g}^{r_{4}}-x_{g}^{r_{5}}\right),
\end{aligned}
$$

which perturbs a random vector $x_{g}^{r_{1}}$ by moving the perturbation at location between the best vector $x_{g}^{\text {best }}$ and a random vector $x_{g}^{r_{1}}$. This perturbation contains five random vectors $x_{g}^{r_{1}}$, $x_{g}^{r_{2}}, x_{g}^{r_{3}}, x_{g}^{r_{4}}, x_{g}^{r_{5}}$ and the best vector $x_{g}^{\text {best }}$ with two weighted difference vectors.

$\left(\mathrm{v}_{22}\right) \mathrm{DE} /$ rand to current/2 mutation strategy was used by Elsayed et $\mathrm{al}^{58,59}$. This mutation strategy has no conflict with the other mutation strategies in the literature. The equation is

$$
v_{g}^{i}=x_{g}^{r_{1}}+F\left(x_{g}^{r_{2}}-x_{g}^{i}\right)+F\left(x_{g}^{r_{3}}-x_{g}^{r_{4}}\right),
$$

which perturbs a random vector $x_{g}^{r_{1}}$ by moving its perturbation location towards location between a random vector $x_{g}^{r_{2}}$ and the current vector $x_{g}^{i}$. This perturbation contains four random vectors $x_{g}^{r_{1}}, x_{g}^{r_{2}}, x_{g}^{r_{3}}, x_{g}^{r_{4}}$ and the current vector $x_{g}^{i}$ with one difference vector.

$\left(\mathrm{v}_{23}\right) \mathrm{DE} / \mathrm{rand}$ to best\&current/2 mutation strategy was used by Elsayed et $\mathrm{al}^{58,59}$. This mutation strategy has no conflict with other mutation strategies in the literature. The equation is

$$
v_{g}^{i}=x_{g}^{r_{1}}+F\left(x_{g}^{\text {best }}-x_{g}^{r_{2}}\right)+F\left(x_{g}^{r_{3}}-x_{g}^{i}\right),
$$

which perturbs a random vector $x_{g}^{r_{1}}$ by moving its perturbation location towards location between the best vector $x_{g}^{\text {best }}$ and random vector $x_{g}^{r_{2}}$. This perturbation contains three random vectors $x_{g}^{r_{1}}, x_{g}^{r_{2}}, x_{g}^{r_{3}}$, the current vector $x_{g}^{i}$ and a best vector $x_{g}^{\text {best }}{ }^{8}$ with a difference vector that utilizes the current vector.

$\left(\mathrm{v}_{24}\right) \mathrm{DE} / \mathrm{mid}$ to better/1 mutation strategy was used by Xin et $\mathrm{al}^{36}$ for designing powerful optimizer. This mutation strategy has no conflict with the other mutation strategies in the literature. The equation is

$$
\begin{aligned}
v_{g}^{i}=\frac{1}{2} & F\left(x_{g}^{\text {bettle }}+x_{g}^{i}\right) \\
& +F\left(x_{g}^{\text {bettle }}-x_{g}^{i}\right)+F\left(x_{g}^{r_{1}}-x_{g}^{r_{2}}\right),
\end{aligned}
$$

which perturbs the average of a better vector $x_{g}^{\text {better }}$ and the current vector $x_{g}^{i}$ by moving its $\stackrel{g}{\text { perturbation location towards location between }}$ a better vector $x_{g}^{\text {better }}$ and the current vector $x_{g}^{i}$. This perturbation contains two random vectors $x_{g}^{r_{1}}, x_{g}^{r_{2}}$, the current vector $x_{g}^{i}$ and a better vector $x_{g}^{\text {better }}$ with one weighted difference vector. This variant is different from other variants in the sense that it only uses a better vector along with average of a better vector and the current vector.

$\left(v_{25}\right) \mathrm{DE} / \mathrm{rand} / 3$ mutation strategy was used by Elsayed et $\mathrm{al}^{58,59}$. This mutation strategy has no conflict with the other mutation strategies in literature. The equation is

$$
\begin{aligned}
v_{g}^{i}=x_{g}^{r_{1}} & +F\left(x_{g}^{r_{2}}-x_{g}^{r_{3}}\right) \\
& +F\left(x_{g}^{r_{4}}-x_{g}^{r_{5}}\right)+F\left(x_{g}^{r_{6}}-x_{g}^{r_{7}}\right),
\end{aligned}
$$

which contains seven random vectors $x_{g}^{r_{1}}, x_{g}^{r_{2}}$, $x_{g}^{r_{3}}, x_{g}^{r_{4}}, x_{g}^{r_{5}}, x_{g}^{r_{6}}, x_{g}^{r_{7}}$ in perturbation of a random vector $x_{g}^{r_{1}}$ with three weighted difference vectors.

$\left(v_{26}\right)$ DE/best/3 mutation strategy was used by Elsayed et $\mathrm{al}^{58,59}$. This mutation strategy has no conflict with the other mutation strategies in 
Table 1 List of DE mutation variants available in the literature.

\begin{tabular}{|c|c|c|}
\hline No. & Variant name & Equations \\
\hline $\mathrm{v}_{1}$ & $\mathrm{DE} /$ rand/1 & $v_{g}^{i}=x_{g}^{r_{1}}+F\left(x_{g}^{r_{2}}-x_{g}^{r_{3}}\right)$ \\
\hline $\mathrm{v}_{2}$ & $\mathrm{DE} /$ best/1 & $v_{g}^{i}=x_{g}^{\text {best }}+F\left(x_{g}^{r_{2}}-x_{g}^{r_{3}}\right)$ \\
\hline$v_{3}$ & $\mathrm{DE} / \mathrm{rand} / 2$ & $v_{g}^{i}=x_{g}^{r_{1}}+F\left(x_{g}^{r_{2}}-x_{g}^{r_{3}}\right)+F\left(x_{g}^{r_{4}}-x_{g}^{r_{5}}\right)$ \\
\hline $\mathrm{v}_{4}$ & $\mathrm{DE} /$ best/2 & $v_{g}^{i}=x_{g}^{\text {best }}+F\left(x_{g}^{r_{1}}-x_{g}^{r_{2}}\right)+F\left(x_{g}^{r_{3}}-x_{g}^{r_{4}}\right)$ \\
\hline$v_{5}$ & $\mathrm{DE} /$ current to rand/1 & $v_{g}^{i}=x_{g}^{i}+F\left(x_{g}^{r_{1}}-x_{g}^{i}\right)+F\left(x_{g}^{r_{2}}-x_{g}^{r_{3}}\right)$ \\
\hline $\mathrm{v}_{6}$ & $\mathrm{DE} /$ current to rand/1 & $v_{g}^{i}=x_{g}^{r_{1}}+F\left(x_{g}^{r_{2}}-x_{g}^{i}\right)+F\left(x_{g}^{r_{1}}-x_{g}^{r_{3}}\right)$ \\
\hline $\mathrm{v}_{7}$ & $\mathrm{DE} /$ current to best/ 1 & $v_{g}^{i}=x_{g}^{i}+F\left(x_{g}^{\text {best }}-x_{g}^{i}\right)+F\left(x_{g}^{r_{1}}-x_{g}^{r_{2}}\right)$ \\
\hline $\mathrm{v}_{8}$ & $\mathrm{DE} /$ current to best/ 1 & $v_{g}^{i}=x_{g}^{i}+F\left(x_{g}^{\text {best }}-x_{g}^{r_{1}}\right)+F\left(x_{g}^{r_{1}}-x_{g}^{r_{2}}\right)$ \\
\hline$v_{9}$ & $\mathrm{DE} /$ rand to best/1 & $v_{g}^{i}=x_{g}^{i}+F\left(x_{g}^{\text {best }}-x_{g}^{r_{1}}\right)+F\left(x_{g}^{r_{1}}-x_{g}^{r_{2}}\right)$ \\
\hline $\mathrm{v}_{10}$ & $\mathrm{DE} /$ rand to best/1 & $v_{g}^{i}=x_{g}^{i}+F\left(x_{g}^{\text {best }}-x_{g}^{i}\right)+F\left(x_{g}^{r_{1}}-x_{g}^{r_{2}}\right)$ \\
\hline $\mathrm{v}_{11}$ & $\mathrm{DE} /$ rand to best/1 & $v_{g}^{i}=x_{g}^{r_{1}}+F\left(x_{g}^{\text {best }}-x_{g}^{r_{2}}\right)+F\left(x_{g}^{r_{3}}-x_{g}^{r_{4}}\right)$ \\
\hline $\mathrm{v}_{12}$ & $\mathrm{DE} /$ rand to best/1 & $v_{g}^{i}=x_{g}^{r_{1}}+F\left(x_{g}^{\text {best }}-x_{g}^{r_{1}}\right)+F\left(x_{g}^{r_{2}}-x_{g}^{r_{3}}\right)$ \\
\hline $\mathrm{v}_{13}$ & $\mathrm{DE} /$ rand to best/1 & $v_{g}^{i}=x_{g}^{r_{1}}+F\left(x_{g}^{\text {best }}-x_{g}^{i}\right)+F\left(x_{g}^{r_{2}}-x_{g}^{r_{3}}\right)$ \\
\hline $\mathrm{v}_{14}$ & $\mathrm{DE} /$ current to best/ 2 & $v_{g}^{i}=x_{g}^{i}+F\left(x_{g}^{\text {best }}-x_{g}^{i}\right)+F\left(x_{g}^{r_{1}}-x_{g}^{r_{2}}\right)$ \\
\hline $\mathrm{v}_{15}$ & $\mathrm{DE} /$ current to best/ 2 & $v_{g}^{i}=x_{g}^{i}+F\left(x_{g}^{\text {best }}-x_{g}^{i}\right)+F\left(x_{g}^{r_{1}}-x_{g}^{r_{2}}\right)+F\left(x_{g}^{r_{3}}-x_{g}^{r_{4}}\right)$ \\
\hline $\mathrm{v}_{16}$ & $\mathrm{DE} /$ current to rand/2 & $v_{g}^{i}=x_{g}^{i}+F\left(x_{g}^{r_{1}}-x_{g}^{i}\right)+F\left(x_{g}^{r_{2}}-x_{g}^{r_{3}}\right)+F\left(x_{g}^{r_{4}}-x_{g}^{r_{5}}\right)$ \\
\hline $\mathrm{v}_{17}$ & $\mathrm{DE} /$ rand to best/ 2 & $v_{g}^{i}=x_{g}^{r_{1}}+F\left(x_{g}^{\text {best }}-x_{g}^{r_{2}}\right)+F\left(x_{g}^{r_{3}}-x_{g}^{r_{4}}\right)$ \\
\hline $\mathrm{v}_{18}$ & $\mathrm{DE} /$ rand to best/ 2 & $v_{g}^{i}=x_{g}^{i}+F\left(x_{g}^{\text {best }}-x_{g}^{i}\right)+F\left(x_{g}^{r_{1}}-x_{g}^{r_{2}}\right)$ \\
\hline $\mathrm{V}_{19}$ & $\mathrm{DE} /$ rand to best/ 2 & $v_{g}^{i}=x_{g}^{r_{1}}+F\left(x_{g}^{\text {best }}-x_{g}^{i}\right)+F\left(x_{g}^{r_{2}}-x_{g}^{r_{3}}\right)+F\left(x_{g}^{r_{4}}-x_{g}^{r_{5}}\right)$ \\
\hline $\mathrm{v}_{20}$ & $\mathrm{DE} /$ rand to best/2 & $v_{g}^{i}=x_{g}^{i}+F\left(x_{g}^{\text {best }}-x_{g}^{i}\right)+F\left(x_{g}^{r_{1}}-x_{g}^{r_{2}}\right)+F\left(x_{g}^{r_{3}}-x_{g}^{r_{4}}\right)$ \\
\hline $\mathrm{v}_{21}$ & $\mathrm{DE} /$ rand to best/ 2 & $v_{g}^{i}=x_{g}^{r_{1}}+F\left(x_{g}^{\text {best }}-x_{g}^{r_{1}}\right)+F\left(x_{g}^{r_{2}}-x_{g}^{r_{3}}\right)+F\left(x_{g}^{r_{4}}-x_{g}^{r_{5}}\right)$ \\
\hline $\mathrm{v}_{22}$ & $\mathrm{DE} /$ rand to current/2 & $v_{g}^{i}=x_{g}^{r_{1}}+F\left(x_{g}^{r_{2}}-x_{g}^{i}\right)+F\left(x_{g}^{r_{3}}-x_{g}^{r_{4}}\right)$ \\
\hline $\mathrm{v}_{23}$ & $\mathrm{DE} /$ rand to best\&current/2 & $v_{g}^{i}=x_{g}^{r_{1}}+F\left(x_{g}^{\text {best }}-x_{g}^{r_{2}}\right)+F\left(x_{g}^{r_{3}}-x_{g}^{i}\right)$ \\
\hline $\mathrm{v}_{24}$ & $\mathrm{DE} / \mathrm{mid}$ to better $/ 1$ & $v_{g}^{i}=\frac{1}{2} F\left(x_{g}^{\text {bettle }}+x_{g}^{i}\right)+F\left(x_{g}^{\text {bettle }}-x_{g}^{i}\right)+F\left(x_{g}^{r_{1}}-x_{g}^{r_{2}}\right)$ \\
\hline $\mathrm{v}_{25}$ & $\mathrm{DE} /$ rand/3 & $v_{g}^{i}=x_{g}^{r_{1}}+F\left(x_{g}^{r_{2}}-x_{g}^{r_{3}}\right)+F\left(x_{g}^{r_{4}}-x_{g}^{r_{5}}\right)+F\left(x_{g}^{r_{6}}-x_{g}^{r_{7}}\right)$ \\
\hline $\mathrm{v}_{26}$ & $\mathrm{DE} /$ best/3 & $v_{g}^{i}=x_{g}^{\text {best }}+F\left(x_{g}^{r_{1}}-x_{g}^{r_{2}}\right)+F\left(x_{g}^{r_{3}}-x_{g}^{r_{4}}\right)+F\left(x_{g}^{r_{5}}-x_{g}^{r_{6}}\right)$ \\
\hline
\end{tabular}

literature. The equation is

$$
\begin{aligned}
v_{g}^{i}=x_{g}^{\text {best }} & +F\left(x_{g}^{r_{1}}-x_{g}^{r_{2}}\right) \\
& +F\left(x_{g}^{r_{3}}-x_{g}^{r_{4}}\right)+F\left(x_{g}^{r_{5}}-x_{g}^{r_{6}}\right),
\end{aligned}
$$

which contains the best vector $x_{g}^{\text {best }}$ and six random vectors $x_{g}^{r_{1}}, x_{g}^{r_{2}}, x_{g}^{r_{3}}, x_{g}^{r_{4}}, x_{g}^{r_{5}}, x_{g}^{r_{6}}$ in perturbation of the best vector $x_{g}^{\text {best }}$ with three weighted difference vectors.

DE is an emerging evolutionary algorithm; it contains a number of mutation variants/strategies. Various mutation strategies in the literature restrain irregularities with respect to naming of the variants and mathematical equation of variants. DE algorithm variants should be misinterpretation-free for its prosperity as an algorithm; to make this algorithm problem-free, an effort is made in this study to identify and remove the inconsistencies associated with DE algorithm variants in the literature. The detailed variants in terms of binomial and exponential schemes are reported in Table 1.

Inconsistencies in $\mathrm{DE}$ mutation strategies are identified with respect to their names and equations. Mathematical equations of variants have a key role since the implementation of each variant is carried out according to its mathematical equations.

\section{IDENTIFICATION OF VARIANTS MATHEMATICAL EQUATION INCONSISTENCIES}

Numerous variants in the literature contain the same mathematical equations but different names, which creates inconsistencies and lead to false impression. In this section, variants of Table 1 having the same mathematical equations and different 
names are identified.

Variants $v_{8}$ and $v_{9}$ have the same mathematical equation but different names, DE/current to best/ 1 and $D E /$ rand to best/1, respectively.

Variants $\mathrm{v}_{7}, \mathrm{v}_{10}, \mathrm{v}_{14}$, and $\mathrm{v}_{18}$ have the same mathematical equations but different names, $\mathrm{DE} /$ current to best/1, DE/rand to best/ 1 , DE/current to best $/ 2$, and $\mathrm{DE} / \mathrm{rand}$ to best $/ 2$, respectively.

Variants $\mathrm{v}_{11}$ and $\mathrm{v}_{17}$ have the same mathematical equations but different names, DE/rand to best/ 1 and $\mathrm{DE} /$ rand to best/2, respectively.

Variants $\mathrm{v}_{15}$ and $\mathrm{v}_{20}$ have the same mathematical equations but different names, $\mathrm{DE} /$ current to best/ 2 and $\mathrm{DE} /$ rand to best/2, respectively.

\section{IDENTIFICATION OF VARIANTS NAMING INCONSISTENCIES}

There are many variants in the literature having the same name but different mathematical equations that fabricate misunderstanding for researchers.

Two variants $v_{5}$ and $v_{6}$ have the same name $\mathrm{DE} /$ current to rand/1 but different mathematical equations.

Two variants $v_{7}$ and $v_{8}$ have the same name $\mathrm{DE} /$ current to best/1 but different equations.

Five variants $v_{9}-v_{13}$ have the same name $\mathrm{DE} / \mathrm{rand}$ to best/1 but different equations.

Two variants $\mathrm{v}_{14}$ and $\mathrm{v}_{15}$ have the same name $\mathrm{DE} /$ current to best/2 but different equations.

Five variants $\mathrm{v}_{17}-\mathrm{v}_{21}$ have the same name $\mathrm{DE} / \mathrm{rand}$ to best/ 2 but different equations.

\section{THE PROPOSED SCHEME TO REMOVE NAMING AND EQUATION INCONSISTENCIES}

The variant having the same mathematical equations but different names are combined because variant having the same equation produced the same representation and the same results that create problem for the users due to names. Variants having the same equations and different names are combined and reported in Table 2 ; variants $\mathrm{v}_{8}$ and $\mathrm{v}_{9}$ are combined as $\mathrm{V}_{8}$ because they have the same mathematical equation, variants $\mathrm{v}_{7}, \mathrm{v}_{10}, \mathrm{v}_{14}$ and $\mathrm{v}_{18}$ are combined as $\mathrm{V}_{7}$, variants $\mathrm{v}_{11}$ and $\mathrm{v}_{17}$ are combined as $\mathrm{V}_{9}$, and variants $\mathrm{v}_{15}$ and $\mathrm{v}_{20}$ are combined as $\mathrm{V}_{12}$. After combing these variants, they have assigned unique names refereeing the equation of that variant.

The naming inconsistency is removed by suggesting the consistent names and reported as suggested names in Table 2 . The suggested names are relative to the equation of variant by considering number of vectors, the based vector and the arrangement of vectors used to form the equation of variant.

The variant $\mathrm{V}_{5}$ has the same name as of variant $V_{6}, D E /$ current to rand/1. $V_{5}$ has equation (9) that places the perturbation at a location between the current population member and a randomly chosen population member. One weighted difference vector is used in the equation of the variant. The equation of $\mathrm{V}_{5}$ confirms the name of the variant $\mathrm{DE} /$ current to rand/1. However, $\mathrm{V}_{6}$ has equation (10) that places the perturbation at a location away from a random vector $x_{g}^{r_{1}}$ between the current vector and another random vector. This scheme contains three random vectors $x_{g}^{r_{1}}, x_{g}^{r_{2}}, x_{g}^{r_{3}}$ and the current vector $x_{g}^{i}$ in the perturbation by repeating a random number $x_{g}^{r_{1}}$ in the weighted difference vector. The suitable name of this variant relative to its equation is $\mathrm{DE} / \mathrm{rand}$ repeat\&current to rand/1, because a based random vector $x_{g}^{r_{1}}$ is repeated in the weighted difference vector and the perturbation location is between the current vector and a random vector.

The variants $\mathrm{V}_{7}-\mathrm{V}_{11}$ have naming inconsistency with each other. Variant $V_{7}$ is used in the literature with various names, $\mathrm{DE} /$ current to best/1, DE/rand to best/1, DE/current to best/2, and $D E / r$ and to best/2. $V_{7}$ has equation (11) that places the perturbation at a location between the current vector $x_{g}^{i}$ and the best vector $x_{g}^{\text {best }}$ at the current generation. The variant uses one weighted difference vector of random vectors $x_{g}^{r_{1}}, x_{g}^{r_{2}}$ in perturbing the current vector $x_{g}^{i}$. The suitable name for this variant relative to its equation is $\mathrm{DE} /$ current to best/1.

Variant $\mathrm{V}_{8}$ has names $\mathrm{DE} /$ current to best/ 1 and DE/rand to best/ 1 in the literature and has equation (12), which perturbs the current vector $x_{g}^{i}$ by moving perturbation at a location between the best vector $x_{g}^{\text {best }}$ and a random vector $x_{g}^{r_{1}}$. The variant uses two random vectors $x_{g}^{r_{1}}, x_{g}^{r_{2}}$, the best vector $x_{g}^{\text {best }}$ and the current vector $x_{g}^{i}$ in perturbation with one weighted difference vector by repeating a random vector $x_{g}^{r_{1}}$. The suitable name of this variant relative to its equation is $\mathrm{DE} /$ current\&rand repeat to best/1.

Variant $\mathrm{V}_{9}$ has names $\mathrm{DE} / \mathrm{rand}$ to best/1 and $\mathrm{DE} / \mathrm{rand}$ to best/ 2 in the literature and has equation (15), which perturbs a random vector $x_{g}^{r_{1}}$ by moving perturbation at a location between best vector $x_{g}^{\text {best }}$ and a random vector $x_{g}^{r_{1}}$. This perturbation uses four random vectors $x_{g}^{r_{1}}, x_{g}^{r_{2}}, x_{g}^{r_{3}}$, $x_{g}^{r_{4}}$ and the best vector with one weighted difference 
Table 2 List of DE variants after removing equation and name inconsistencies.

\begin{tabular}{|c|c|c|c|}
\hline No. & Variant name(s) & Equations & Suggested name ${ }^{\dagger}$ \\
\hline $\mathrm{V}_{1}$ & $\mathrm{DE} / \mathrm{rand} / 1$ & $v_{g}^{i}=x_{g}^{r_{1}}+F\left(x_{g}^{r_{2}}-x_{g}^{r_{3}}\right)$ & \\
\hline $\mathrm{V}_{2}$ & $\mathrm{DE} /$ best/1 & $v_{g}^{i}=x_{g}^{\text {best }}+F\left(x_{g}^{r_{2}}-x_{g}^{r_{3}}\right)$ & \\
\hline $\mathrm{V}_{3}$ & $\mathrm{DE} / \mathrm{rand} / 2$ & $v_{g}^{i}=x_{g}^{r_{1}}+F\left(x_{g}^{r_{2}}-x_{g}^{r_{3}}\right)+F\left(x_{g}^{r_{4}}-x_{g}^{r_{5}}\right)$ & \\
\hline $\mathrm{V}_{4}$ & $\mathrm{DE} /$ best/2 & $v_{g}^{i}=x_{g}^{\text {best }}+F\left(x_{g}^{r_{1}}-x_{g}^{r_{2}}\right)+F\left(x_{g}^{r_{3}}-x_{g}^{r_{4}}\right)$ & \\
\hline $\mathrm{V}_{5}$ & $\mathrm{DE} /$ current to rand/1 & $v_{g}^{i}=x_{g}^{i}+F\left(x_{g}^{r_{1}}-x_{g}^{i}\right)+F\left(x_{g}^{r_{2}}-x_{g}^{r_{3}}\right)$ & $\mathrm{DE} /$ current to rand/1 \\
\hline $\mathrm{V}_{6}$ & $\mathrm{DE} /$ current to rand/1 & $v_{g}^{i}=x_{g}^{r_{1}}+F\left(x_{g}^{r_{2}}-x_{g}^{i}\right)+F\left(x_{g}^{r_{1}}-x_{g}^{r_{3}}\right)$ & $\mathrm{DE} /$ rand repeat\&current to rand/ 1 \\
\hline $\mathrm{V}_{7}$ & $\left\{\begin{array}{l}\mathrm{DE} / \text { current to best/1 } \\
\mathrm{DE} / \text { rand to best/1 } \\
\mathrm{DE} / \text { current to best/2 } \\
\mathrm{DE} / \text { rand to best/2 }\end{array}\right.$ & $v_{g}^{i}=x_{g}^{i}+F\left(x_{g}^{\text {best }}-x_{g}^{i}\right)+F\left(x_{g}^{r_{1}}-x_{g}^{r_{2}}\right)$ & $\mathrm{DE} /$ current to best/1 \\
\hline $\mathrm{V}_{8}$ & $\left\{\begin{array}{l}\mathrm{DE} / \text { current to best/1 } \\
\mathrm{DE} / \text { rand to best/1 }\end{array}\right.$ & $v_{g}^{i}=x_{g}^{i}+F\left(x_{g}^{\text {best }}-x_{g}^{r_{1}}\right)+F\left(x_{g}^{r_{1}}-x_{g}^{r_{2}}\right)$ & $\mathrm{DE} /$ current\&rand repeat to best/ 1 \\
\hline $\mathrm{V}_{9}$ & $\left\{\begin{array}{l}\mathrm{DE} / \mathrm{rand} \text { to best } / 1 \\
\mathrm{DE} / \mathrm{rand} \text { to best/2 }\end{array}\right.$ & $v_{g}^{i}=x_{g}^{r_{1}}+F\left(x_{g}^{\text {best }}-x_{g}^{r_{2}}\right)+F\left(x_{g}^{r_{3}}-x_{g}^{r_{4}}\right)$ & $\mathrm{DE} /$ rand to best/1 \\
\hline $\mathrm{V}_{10}$ & $\mathrm{DE} /$ rand to best/1 & $v_{g}^{i}=x_{g}^{r_{1}}+F\left(x_{g}^{\text {best }}-x_{g}^{r_{1}}\right)+F\left(x_{g}^{r_{2}}-x_{g}^{r_{3}}\right)$ & $\mathrm{DE} /$ rand repeat to best/ 1 \\
\hline $\mathrm{V}_{11}$ & $\mathrm{DE} /$ rand to best/1 & $v_{g}^{i}=x_{g}^{r_{1}}+F\left(x_{g}^{\text {best }}-x_{g}^{i}\right)+F\left(x_{g}^{r_{2}}-x_{g}^{r_{3}}\right)$ & $\mathrm{DE} /$ rand\&current to best/ 1 \\
\hline $\mathrm{V}_{12}$ & $\left\{\begin{array}{l}\mathrm{DE} / \text { current to best } / 2 \\
\mathrm{DE} / \text { rand to best } / 2\end{array}\right.$ & $\begin{aligned} v_{g}^{i} & =x_{g}^{i}+F\left(x_{g}^{\text {best }}-x_{g}^{i}\right) \\
& +F\left(x_{g}^{r_{1}}-x_{g}^{r_{2}}\right)+F\left(x_{g}^{r_{3}}-x_{g}^{r_{4}}\right)\end{aligned}$ & $\mathrm{DE} /$ current to best/2 \\
\hline $\mathrm{V}_{13}$ & $\mathrm{DE} /$ current to rand/2 & $\begin{aligned} v_{g}^{i} & =x_{g}^{i}+F\left(x_{g}^{r_{1}}-x_{g}^{i}\right) \\
& +F\left(x_{g}^{r_{2}}-x_{g}^{r_{3}}\right)+F\left(x_{g}^{r_{4}}-x_{g}^{r_{5}}\right)\end{aligned}$ & \\
\hline $\mathrm{V}_{14}$ & $\mathrm{DE} /$ rand to best $/ 2$ & $\begin{aligned} v_{g}^{i} & =x_{g}^{r_{1}}+F\left(x_{g}^{\text {best }}-x_{g}^{i}\right) \\
& +F\left(x_{g}^{r_{2}}-x_{g}^{r_{3}}\right)+F\left(x_{g}^{r_{4}}-x_{g}^{r_{5}}\right)\end{aligned}$ & $\mathrm{DE} /$ rand\&current to best $/ 2$ \\
\hline $\mathrm{V}_{15}$ & $\mathrm{DE} /$ rand to best/2 & $\begin{aligned} v_{g}^{i} & =x_{g}^{r_{1}}+F\left(x_{g}^{\text {best }}-x_{g}^{r_{1}}\right) \\
& +F\left(x_{g}^{r_{2}}-x_{g}^{r_{3}}\right)+F\left(x_{g}^{r_{4}}-x_{g}^{r_{5}}\right)\end{aligned}$ & $\mathrm{DE} /$ rand repeat to best $/ 2$ \\
\hline $\mathrm{V}_{16}$ & $\mathrm{DE} /$ rand to current $/ 2$ & $v_{g}^{i}=x_{g}^{r_{1}}+F\left(x_{g}^{r_{2}}-x_{g}^{i}\right)+F\left(x_{g}^{r_{3}}-x_{g}^{r_{4}}\right)$ & $\mathrm{DE} /$ rand\&current to rand/1 \\
\hline $\mathrm{V}_{17}$ & $\mathrm{DE} /$ rand to best\&current $/ 2$ & $v_{g}^{i}=x_{g}^{r_{1}}+F\left(x_{g}^{\text {best }}-x_{g}^{r_{2}}\right)+F\left(x_{g}^{r_{3}}-x_{g}^{i}\right)$ & $\mathrm{DE} /$ rand to best\&current/ 1 \\
\hline $\mathrm{V}_{18}$ & $\mathrm{DE} / \mathrm{mid}$ to better $/ 1$ & $\begin{aligned} v_{g}^{i} & =\frac{1}{2} F\left(x_{g}^{\text {bettle }}+x_{g}^{i}\right) \\
& +F\left(x_{g}^{\text {bettle }}-x_{g}^{i}\right)+F\left(x_{g}^{r_{1}}-x_{g}^{r_{2}}\right)\end{aligned}$ & \\
\hline $\mathrm{V}_{19}$ & $\mathrm{DE} / \mathrm{rand} / 3$ & $\begin{aligned} v_{g}^{i} & =x_{g}^{r_{1}}+F\left(x_{g}^{r_{2}}-x_{g}^{r_{3}}\right) \\
& +F\left(x_{g}^{r_{4}}-x_{g}^{r_{5}}\right)+F\left(x_{g}^{r_{6}}-x_{g}^{r_{7}}\right)\end{aligned}$ & \\
\hline $\mathrm{V}_{20}$ & $\mathrm{DE} /$ best/3 & $\begin{aligned} v_{g}^{i} & =x_{g}^{\text {best }}+F\left(x_{g}^{r_{1}}-x_{g}^{r_{2}}\right) \\
& +F\left(x_{g}^{r_{3}}-x_{g}^{r_{4}}\right)+F\left(x_{g}^{r_{5}}-x_{g}^{r_{6}}\right)\end{aligned}$ & \\
\hline
\end{tabular}

$\dagger$ Variants with name inconsistencies are suggested with consistent name.

vector. The suitable name of this variant relative to its equation is $\mathrm{DE} /$ rand to best/1.

Both variants $\mathrm{V}_{10}$ and $\mathrm{V}_{11}$ have the name $\mathrm{DE} /$ rand to best/1 in literature, which is the same as $V_{9}$. $V_{10}$ has equation (16) that perturbs a random vector $x_{g}^{r_{1}}$ by moving perturbation at a location between $x_{g}^{r_{1}}$ and the best vector $x_{g}^{\text {best }}$. This perturbation contains three random vectors $x_{g}^{r_{1}}, x_{g}^{r_{2}}$, $x_{g}^{r_{3}}$ and the best vector $x_{g}^{\text {best }}$ with one weighted difference vector. The suggested name of this vari- ant relative to its equation is $\mathrm{DE} /$ rand repeat to best/1.

$\mathrm{V}_{11}$ has equation (17) that perturbs a random vector $x_{g}^{r_{1}}$ by moving perturbation at a location between the best vector $x_{g}^{\text {best }}$ and the current vector $x_{g}^{i}$. This perturbation contains three random vectors $x_{g}^{r_{1}}, x_{g}^{r_{2}}, x_{g}^{r_{3}}$, the current vector $x_{g}^{r_{1}}$ and the best vector $x_{g}^{\text {bes }}$ with one weighted difference vector. The suggested name of this variant relative to its equation is $\mathrm{DE} /$ rand\&current to best/1. 
The variants $\mathrm{V}_{12}, \mathrm{~V}_{14}, \mathrm{~V}_{15}$ have naming inconsistency with one another. $\mathrm{V}_{12}$ in the literature has names $\mathrm{DE} /$ current to best/2 and $\mathrm{DE} / \mathrm{rand}$ to best/2 and has equation (19), which perturbs the current vector $x_{g}^{i}$ by moving perturbation at a location between the best vector $x_{g}^{\text {best }}$ and the current vector. This perturbation contains four random vectors $x_{g}^{r_{1}}, x_{g}^{r_{2}}, x_{g}^{r_{3}}, x_{g}^{r_{4}}$, the best vector $x_{g}^{\text {best }}$ and the current vector $x_{g}^{i}$ with two weighted difference vectors. The suggested name of this variant relative to its equation is $\mathrm{DE} /$ current to best/ 2 .

In the literature, both variants $\mathrm{V}_{14}$ and $\mathrm{V}_{15}$ have the name $\mathrm{DE} / \mathrm{rand}$ to best/2, which is also used for $\mathrm{V}_{12} . \mathrm{V}_{14}$ has equation (23) that perturbs a random vector $x_{g}^{r_{1}}$ by moving perturbation at a location between the best vector $x_{g}^{\text {best }}$ and the current vector $x_{\sigma}^{i}$. This perturbation contains five random vectors $x_{g}^{r_{1}}, x_{g}^{r_{2}}, x_{g}^{r_{3}}, x_{g}^{r_{4}}, x_{g}^{r_{5}}$, the current vector $x_{g}^{i}$ and the best vector $x_{g}^{\text {best }}{ }^{8}$ with two weighted difference vectors. The suggested name of this variant relative to its equation is $\mathrm{DE} /$ rand\&current to best/2.

$\mathrm{V}_{15}$ has equation (25) that perturbs a random vector $x_{g}^{r_{1}}$ by moving perturbation at location between the best vector $x_{g}^{\text {best }}$ and a random vector $x_{o}^{r_{1}}$. This perturbation contains five random vectors $x_{g}^{g}, x_{g}^{r_{2}}, x_{g}^{r_{3}}, x_{g}^{r_{4}}, x_{g}^{r_{5}}$ and the best vector $x_{g}^{\text {best }}$ with two weighted difference vectors. The suitable name of this variant relative to its equation is $\mathrm{DE} / \mathrm{rand}$ repeat to best $/ 2$.

The variant $\mathrm{V}_{16}$ with name $\mathrm{DE} / \mathrm{rand}$ to current/ 2 in the literature has equation (26) that perturbs a random vector $x_{g}^{r_{1}}$ by moving perturbation at a location between a random vector $x_{\sigma}^{r_{2}}$ and the current vector $x_{g}^{i}$. This perturbation contains four random vectors $x_{g}^{r_{1}}, x_{g}^{r_{2}}, x_{g}^{r_{3}}, x_{g}^{r_{4}}$ and the current vector $x_{g}^{i}$ with one difference vector. The suggested name of this variant relative to its equation is $\mathrm{DE} /$ rand\&current to rand/1.

The variant $\mathrm{V}_{17}$ with name $\mathrm{DE} / \mathrm{rand}$ to best\&current/ 2 in literature has equation (27) that perturbs a random vector $x_{g}^{r_{1}}$ by moving perturbation at a location between the best vector $x_{g}^{\text {best }}$ and a random vector $x_{g}^{r_{2}}$. This perturbation contains three random vectors $x_{g}^{r_{1}}, x_{g}^{r_{2}}, x_{g}^{r_{3}}$, the current vector $x_{g}^{i}$ and the best vector $x_{g}^{\text {best }}$ with one difference vector that utilizes the current vector $x_{g}^{i}$. The suggested name of this variant relative to its equation is DE/rand to best\&current/1.

To summarize, mathematical equation inconsistencies in DE mutation variants are removed from Table 1 and reported in Table 2, which still contains naming inconsistencies. Inconsistent names are suggested with new names according to the
Table 3 Test suit of benchmark functions.

\begin{tabular}{ll}
\hline Function & Equation \\
\hline$f_{1}$ & $f(x)=\sum_{i=0}^{n} x_{i}^{2}$ \\
$f_{2}$ & $f(x)=\sum_{i=0}^{n}\left(\sum_{j=0}^{i} x_{i}^{2}\right)^{2}$ \\
$f_{3}$ & $f(x)=\sum_{i=1}^{n-1}\left[100\left(x_{i+1}-x_{i}^{2}\right)^{2}+\left(1-x_{i}^{2}\right)\right]$ \\
$f_{4}$ & $f(x)=\sum_{i=1}^{n}\left[\frac{1}{4000} x_{i}^{2}-\prod_{j=1}^{n} \cos \frac{x_{j}}{\sqrt{j}}+1\right]$ \\
$f_{5}$ & $f(x)=-20 \mathrm{e}^{-0.2 \sqrt{\frac{\sum_{i=1}^{n} x_{i}^{2}}{n}}-\mathrm{e}^{\frac{\sum_{i=1}^{n} \cos 2 \pi x_{i}}{n}}+20+\mathrm{e}}$ \\
$f_{6}$ & $f(x)=\sum_{i=1}^{n}\left\lfloor x_{i}+0.5\right\rfloor^{2}$ \\
\hline
\end{tabular}

corresponding equations and reported in Table 2, which are used in the paper to generate results. The short names of DE mutation variants that will be used throughout the remaining paper for binomial variants are $\mathrm{V}_{1}-\mathrm{V}_{20}$ with corresponding $\mathrm{V}_{21}-\mathrm{V}_{40}$ for exponential variants.

\section{EXPERIMENTAL RESULTS AND DISCUSSION}

To illustrate the varying performance of DE mutation strategies, a test suit of benchmark $n$-dimensional functions taken from Ref. 2 is used (Table 3 ). The experimental results of average fitness values of DE mutation strategies are obtained over 30 independent runs, 2000 training iterations, 10 dimensions, population size $N_{\mathrm{p}}=30$, crossover probability $C_{r}=0.5$, and mutation rate $F=0.7$ in the experimentation. The main aim of this section is to show the diverse performance of DE mutation strategies given in Table 2. The DE mutation strategies are used as $\mathrm{V}_{1}-\mathrm{V}_{20}$ for binomial mutation and $\mathrm{V}_{21}-\mathrm{V}_{40}$ for the corresponding exponential mutation.

The convergence graphs are generated showing iterations along the $x$-axis and the performance along the $y$-axis. The variants are from $\mathrm{V}_{1}-\mathrm{V}_{20}$ for binomial variants and $\mathrm{V}_{21}-\mathrm{V}_{40}$ for the exponential version of each corresponding binomial variant. The convergence graphs are also generated using the same experimental setting for each mutation strategy and function. Convergence graphs of two sample functions $f_{1}$ (Fig. 1) and $f_{3}$ (Fig. 2) are given to show the distinct performance of each variant. Convergence graphs show that each DE mutation strategy has its own optimization curve. 

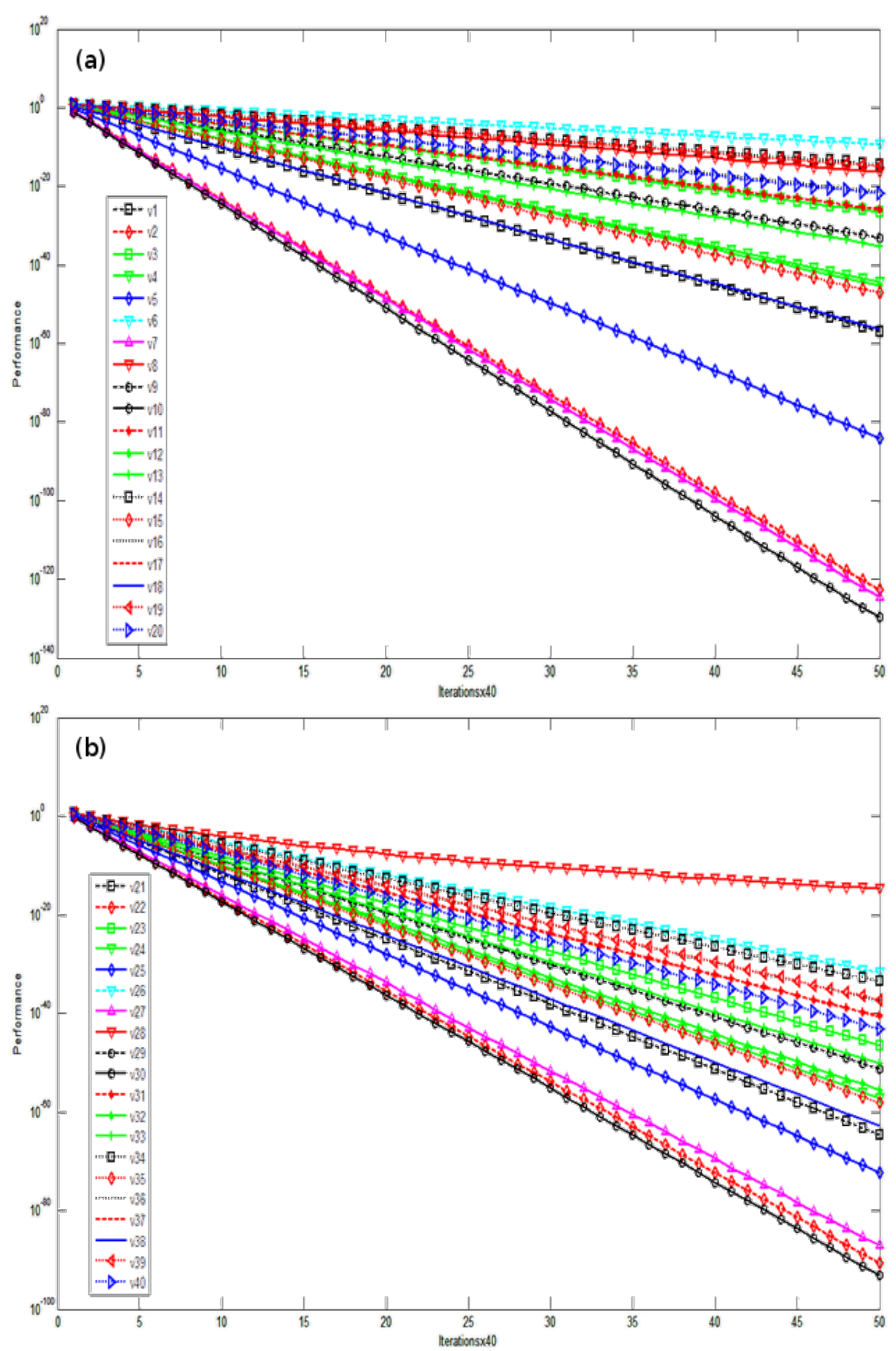

Fig. 1 Logarithmic convergence graphs of average fitness of functions $f_{1}$ for (a) $V_{1}-V_{20}$ and (b) $V_{21}-V_{40}$ showing the average fitness versus the number of iterations.

From experimental results, it is observed that all 40 mutation strategies of $\mathrm{DE}$ algorithm are valid strategies to enhance the functionality of DE algorithm, and hence can be effectively used wherever DE algorithm is used.
The average fitness values of DE mutation strategies for testing suit of functions are given in Table 4. The results are obtained using the same experimental setting for each variant and function. The average fitness results of DE mutation strategies 

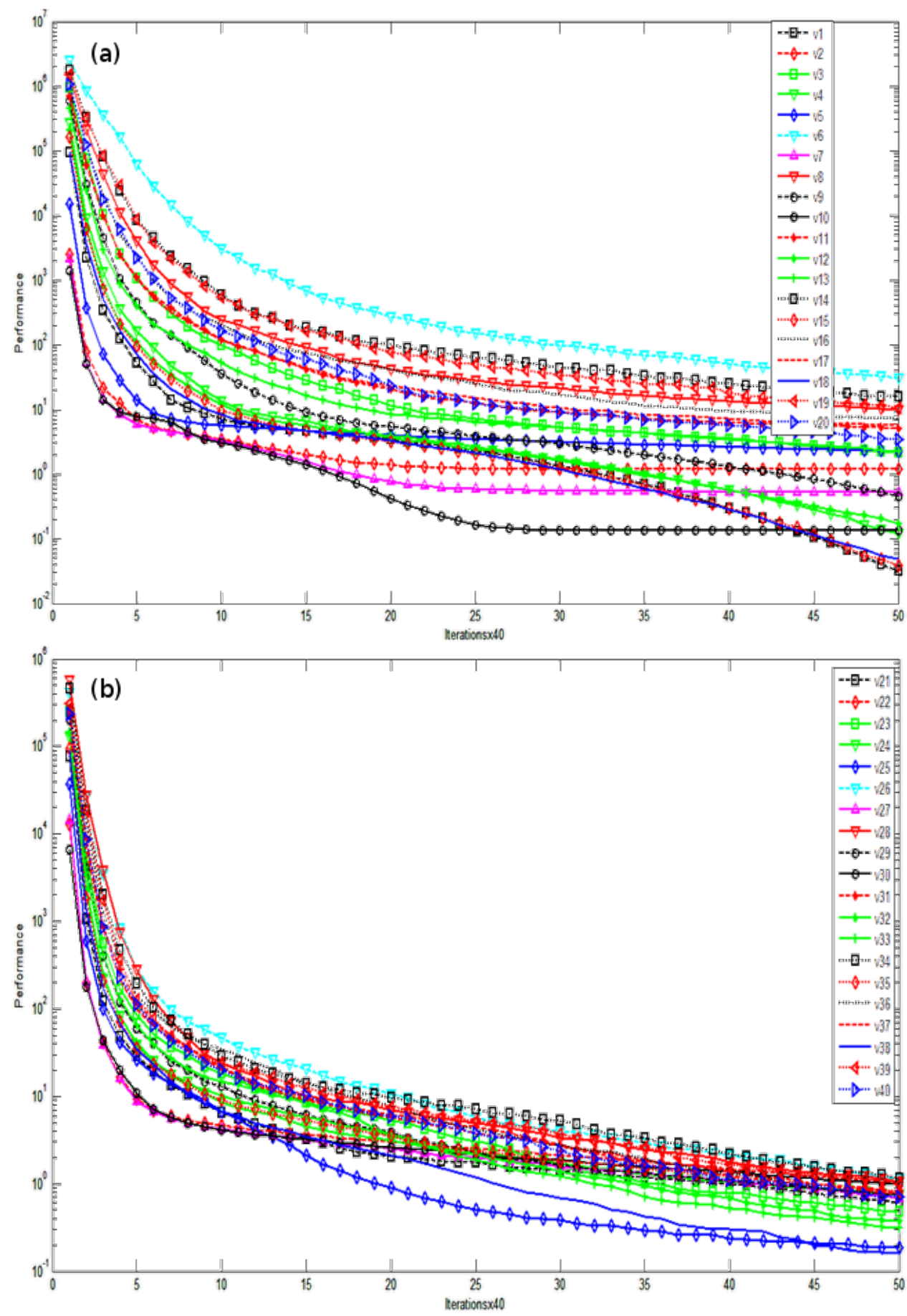

Fig. 2 Logarithmic convergence graphs of average fitness of functions $f_{3}$ for (a) $V_{1}-V_{20}$ and (b) $V_{21}-V_{40}$ showing the average fitness versus the number of iterations.

show distinct performance of each variant for each function.

To explore the diverse performance of DE variants, the ranks of DE variants are calculated. The ranks (Table 5) show that each DE mutation strate- gies have different performance from others. The rank of each variant is calculated based on the of average fitness values of that variant for each function. The values show the corresponding rank of each variant in ascending order; the smaller the rank, the 
Table 4 Average fitness results of DE variants of functions $f_{1}-f_{6}$.

\begin{tabular}{|c|c|c|c|c|c|c|}
\hline Var. & $f_{1}$ & $f_{2}$ & $f_{3}$ & $f_{4}$ & $f_{5}$ & $f_{6}$ \\
\hline $\mathrm{V}_{1}$ & $.3 \pm 2.9) \times 10^{-57}$ & $.2 \pm 4.6) \times 10^{-10}$ & $.1 \pm 1.4) \times 10^{-2}$ & $(2.7 \pm 4.1) \times 10^{-2}$ & $(1.0 \pm 1.4) \times 10^{-1}$ & $(1.5 \pm 1.3) \times 10^{-11}$ \\
\hline $\mathrm{V}_{2}$ & $.8 \pm 8.5) \times 10^{-123}$ & $1.4 \pm 2.3) \times 10^{-32}$ & $1.2 \pm 1.8$ & $5.4 \pm 3.1) \times 10^{-2}$ & $2.7 \pm 0.7) \times 10^{-1}$ & $(0.6 \pm 1.7) \times 10^{-11}$ \\
\hline $\mathrm{V}_{3}$ & $6 \pm 3.3) \times 10^{-27}$ & $7.0 \pm 5.9) \times 10^{-3}$ & $2.1 \pm 0.6$ & $2.7 \pm 0.5) \times 10^{-1}$ & $5.9 \pm 0.6) \times 10^{-1}$ & $(2.1 \pm 2.0) \times 10^{-11}$ \\
\hline $\mathrm{V}_{4}$ & $4 \pm 1.2) \times 10^{-44}$ & $3.7 \pm 5.3) \times 10^{-7}$ & $1.1 \pm 0.8) \times 10^{-1}$ & $2.2 \pm 0.6) \times 10^{-1}$ & $4.3 \pm 1.2) \times 10^{-1}$ & $(2.7 \pm 2.5) \times 10^{-11}$ \\
\hline $\mathrm{V}_{5}$ & $9 \pm 1.3) \times 1($ & $.1 \pm 1.0) \times 10^{-19}$ & $2.2 \pm 1.3$ & $4.5 \pm 3.6) \times 10^{-2}$ & $3.2 \pm 0.5) \times 10^{-1}$ & $(2.4 \pm 2.4) \times 10^{-11}$ \\
\hline $\mathrm{V}_{6}$ & $4 \pm 4.7) \times 10^{-10}$ & $(1.9 \pm 1.3) \times 10^{1}$ & $(3.0 \pm 1.4) \times 10^{1}$ & $3.7 \pm 0.6) \times 10^{-1}$ & x & $(5.6 \pm 5.4) \times 10^{-11}$ \\
\hline $\mathrm{V}_{7}$ & $.7 \pm 9.3) \times 10^{-125}$ & $(0.4 \pm 1.0) \times 10^{-35}$ & $0.5 \pm 1.3$ & $2.8 \pm 1.9) \times 10^{-2}$ & $(2.3 \pm 0.8) \times$ & $(1.3 \pm 1.9) \times 10^{-11}$ \\
\hline $\mathrm{V}_{8}$ & $2 \pm 3.0) \times 10^{-17}$ & $(5.8 \pm 3.3) \times 10^{-3}$ & $9.9 \pm 2.7$ & $3.8 \pm 0.7) \times 10^{-1}$ & $(8.0 \pm 0.8) \times 10^{-1}$ & $(4.2 \pm 4.2) \times 10^{-11}$ \\
\hline $\mathrm{V}_{9}$ & & & $4.5 \pm 1.7) \times$ & $2.5 \pm 0.4) \times 10^{-1}$ & x & (1.8 \\
\hline$V_{10}$ & $0^{-130}$ & $(2.1 \pm 9.9) \times 10^{-32}$ & $1.3 \pm 7.1) \times$ & $(2.1 \pm 1.5) \times 10^{-2}$ & $86 \pm 78) \times$ & $(1.4 \pm 1.8) \times$ \\
\hline$V_{11}$ & -26 & $9.0 \pm 8.5) \times 10^{-2}$ & $5.1 \pm 0.8$ & ה & -1 & $0^{-11}$ \\
\hline$V_{12}$ & & $40+57)$ & $.7 \pm 2.7) \times 10^{-1}$ & $(2.4 \pm 0.5) \times$ & & $9 \pm 39)$ \\
\hline $\mathrm{V}_{13}$ & $0^{-36}$ & $(2.5 \pm 2.1) \times 10^{-5}$ & $2.2 \pm 1.5$ & $(2.5 \pm 0.5) \times 10^{-1}$ & $(5.2 \pm 0.7) \times 10^{-1}$ & $(2.0 \pm 1.9) \times 10^{-11}$ \\
\hline $\mathrm{V}_{14}$ & & $4.5 \pm 1.8$ & & & & $0^{-11}$ \\
\hline 15 & & $.3 \pm 1.5) \times 10^{-7}$ & $7+20) x$ & $(2.2 \pm 0.5) \times$ & $47+05)$ & $(27+40) x$ \\
\hline$V_{16}$ & & & & & & $.0 \pm 2.1)$ \\
\hline$I_{17}$ & & & $5.5 \pm$ & & & \\
\hline $\mathrm{V}_{18}$ & & $(4.5 \pm 5.4) \times 10^{-10}$ & $(4.8 \pm 7.2) \times 10^{-2}$ & $(1.6 \pm 0.5) \times$ & & \\
\hline$v_{19}$ & & $1.2 \pm 0.8$ & $10.0 \pm 7.9$ & & & \\
\hline 20 & & -2 & & & & -11 \\
\hline$V_{21}$ & & & & & & \\
\hline$V_{22}$ & & & & & & )$^{-11}$ \\
\hline $\mathrm{V}_{23}$ & & 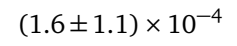 & $(6.7 \pm$ & $(0.3 \pm 1.3) \times$ & & $(63+48)$ \\
\hline $\mathrm{V}_{24}$ & & & & & & \\
\hline$I$ & & & $(3.2 \pm 6.4) \times 10^{-1}$ & & & \\
\hline $\mathrm{V}_{26}$ & $10^{-32}$ & $(2.8 \pm 2.1) \times 10^{-1}$ & $1.1 \pm 0.7$ & $(5.8 \pm 5.3) \times 10^{-3}$ & $(0.4 \pm 2.1) \times$ & $(7.4 \pm 7.3) \times$ \\
\hline$v_{27}$ & & -18 & $(6.9 \pm 9.4) \times 10$ & $(6.5$ & -1 & $(5.0 \pm 6.3) \times$ \\
\hline $\mathrm{V}_{28}$ & & & $(9.9 \pm 8.7) \times$ & & & \\
\hline $\mathrm{V}_{29}$ & & & $(7.2 \pm 6.1) \times$ & & & \\
\hline$V_{30}$ & & $(1.8 \pm 1.8) \times 10^{-17}$ & $0.9 \pm 1.2$ & $(0.8 \pm 2.4) \times 10^{-3}$ & $(0.4 \pm 2.2) \times 10^{-2}$ & $(3.5 \pm 3.5) \times 10^{-11}$ \\
\hline $\mathrm{V}_{31}$ & $2.1 \pm 1.8) \times 10^{-41}$ & $(9.9 \pm 9.6) \times 10^{-3}$ & $0.9 \pm 1.1$ & $(2.3 \pm 3.8) \times 10^{-3}$ & $(7.2 \pm 7.4) \times 10^{-12}$ & $(6.2 \pm 6.3) \times 10^{-11}$ \\
\hline $\mathrm{V}_{32}$ & $5 \pm 2.3) \times 10^{-56}$ & $(0.7 \pm 1.1) \times 10^{-7}$ & $(5.9 \pm 7.0) \times 10^{-1}$ & $(6.8 \pm 5.7) \times 10^{-3}$ & $(0.2 \pm 2.7) \times 1($ & $(3.3 \pm 3.0) \times 10^{-11}$ \\
\hline$V_{33}$ & & $(2.9 \pm 1.9) \times 10^{-6}$ & $(4.0 \pm 2.5) \times 10^{-1}$ & $(6.3 \pm 6.1) \times 10^{-3}$ & $(0.2 \pm 5.4) \times 10$ & $(5.7 \pm 6.2) \times 10^{-11}$ \\
\hline $\mathrm{V}_{34}$ & 4) $\times 10^{-34}$ & $(9.5 \pm 4.8) \times 10^{-2}$ & $(8.3 \pm 5.1) \times 10$ & $(6.4 \pm 5.3) \times 10^{-3}$ & $(1.7 \pm 1.5) \times 10^{-11}$ & $(5.0 \pm 5.0) \times 10^{-11}$ \\
\hline $\mathrm{V}_{35}$ & & $(2.0 \pm 1.8) \times 10^{-7}$ & $(6.1 \pm 6.2) \times 10^{-1}$ & $(0.3 \pm 1.7) \times 10^{-3}$ & $(2.2 \pm 2.1) \times 10^{-12}$ & $(5.6 \pm 5.6) \times 10^{-11}$ \\
\hline $\mathrm{V}_{36}$ & $2.1 \pm 1.7) \times 10^{-38}$ & $(2.9 \pm 1.5) \times 10^{-2}$ & $(7.5 \pm 5.3) \times 10^{-1}$ & $(1.9 \pm 3.0) \times 10^{-3}$ & $(8.8 \pm 9.6) \times 10^{-12}$ & $(0.6 \pm 1.0) \times 10^{-10}$ \\
\hline $\mathrm{V}_{37}$ & & & & & & \\
\hline $\mathrm{V}_{38}$ & & $(1.6 \pm 1.6) \times 10^{-8}$ & $(1.7 \pm 3.1) \times 10^{-1}$ & $(7.8 \pm 6.5) \times 10^{-3}$ & $(7.5 \pm 6.6) \times 10^{-2}$ & $(3.4 \pm 3.4) \times 10^{-11}$ \\
\hline $\mathrm{V}_{39}$ & & & & & & \\
\hline $\mathrm{V}$ & $0.9 \pm 1.1) \times 10^{-43}$ & $(4.8 \pm 5.4) \times 10^{-4}$ & $(8.6 \pm 5.8) \times 10^{-1}$ & $(4.0 \pm 5.3) \times 10^{-3}$ & $(1.6 \pm 4.2) \times 10^{-2}$ & $(7.5 \pm 5.6) \times 10^{-11}$ \\
\hline
\end{tabular}

better performance of the mutation strategy.

\section{CONCLUSIONS}

The selection of a DE variant affects the performance result of DE algorithm since there is a deviation in performance of $\mathrm{DE}$ variants. $\mathrm{DE}$ algorithm variants in the literature have naming and mathematical equation inconsistencies. This study identifies these inconsistencies and presents a consistent set of DE variants. The details of naming and formulation inconsistencies is discussed. The naming and formulation inconsistencies are removed based on the number of vectors and order of vectors used to form the equation of variant. This study will prove to be a significant addition in DE literature in view of the fact that the existence of any inconsistency might trigger off new researchers. Further studies are still required to explore the limitations, advantages, and flaws in this direction. The main focus in this work is not the overwhelming existing DE variants but the new tracks to work on the DE variants in optimization. Future work of this study is to perform a thorough analysis of these $\mathrm{DE}$ variants to access the performance of each DE 
Table 5 Average fitness ranks of DE variants of functions.

\begin{tabular}{lcccccc}
\hline Var. & $f_{1}$ & $f_{2}$ & $f_{3}$ & $f_{4}$ & $f_{5}$ & $f_{6}$ \\
\hline $\mathrm{V}_{1}$ & 13 & 9 & 1 & 22 & 17 & 5 \\
$\mathrm{~V}_{2}$ & 3 & 2 & 28 & 25 & 24 & 1 \\
$\mathrm{~V}_{3}$ & 31 & 27 & 30 & 32 & 33 & 8 \\
$\mathrm{~V}_{4}$ & 21 & 18 & 4 & 27 & 26 & 15 \\
$\mathrm{~V}_{5}$ & 7 & 4 & 32 & 24 & 25 & 11 \\
$\mathrm{~V}_{6}$ & 40 & 40 & 40 & 39 & 40 & 30 \\
$\mathrm{~V}_{7}$ & 2 & 1 & 13 & 23 & 23 & 3 \\
$\mathrm{~V}_{8}$ & 36 & 26 & 37 & 40 & 39 & 25 \\
$\mathrm{~V}_{9}$ & 29 & 24 & 11 & 31 & 31 & 6 \\
$\mathrm{~V}_{10}$ & 1 & 3 & 5 & 19 & 15 & 4 \\
$\mathrm{~V}_{11}$ & 32 & 34 & 34 & 34 & 34 & 18 \\
$\mathrm{~V}_{12}$ & 20 & 13 & 6 & 29 & 29 & 24 \\
$\mathrm{~V}_{13}$ & 27 & 21 & 31 & 30 & 30 & 7 \\
$\mathrm{~V}_{14}$ & 39 & 39 & 39 & 38 & 38 & 17 \\
$\mathrm{~V}_{15}$ & 18 & 15 & 2 & 28 & 28 & 14 \\
$\mathrm{~V}_{16}$ & 35 & 37 & 36 & 35 & 35 & 13 \\
$\mathrm{~V}_{17}$ & 33 & 33 & 35 & 33 & 32 & 12 \\
$\mathrm{~V}_{18}$ & 14 & 10 & 3 & 26 & 27 & 9 \\
$\mathrm{~V}_{19}$ & 37 & 38 & 38 & 37 & 37 & 16 \\
$\mathrm{~V}_{20}$ & 34 & 32 & 33 & 36 & 36 & 10 \\
$\mathrm{~V}_{21}$ & 9 & 11 & 12 & 4 & 11 & 23 \\
$\mathrm{~V}_{22}$ & 5 & 7 & 8 & 20 & 16 & 2 \\
$\mathrm{~V}_{23}$ & 19 & 22 & 16 & 2 & 8 & 37 \\
$\mathrm{~V}_{24}$ & 12 & 17 & 21 & 18 & 13 & 29 \\
$\mathrm{~V}_{25}$ & 8 & 8 & 9 & 10 & 21 & 22 \\
$\mathrm{~V}_{26}$ & 30 & 36 & 27 & 12 & 7 & 38 \\
$\mathrm{~V}_{27}$ & 6 & 5 & 18 & 15 & 18 & 27 \\
$\mathrm{~V}_{28}$ & 38 & 23 & 26 & 21 & 22 & 40 \\
$\mathrm{~V}_{29}$ & 16 & 20 & 19 & 1 & 10 & 33 \\
$\mathrm{~V}_{30}$ & 4 & 6 & 24 & 5 & 8 & 21 \\
$\mathrm{~V}_{31}$ & 23 & 28 & 25 & 8 & 4 & 36 \\
$\mathrm{~V}_{32}$ & 15 & 14 & 14 & 16 & 20 & 19 \\
$\mathrm{~V}_{33}$ & 17 & 19 & 10 & 13 & 19 & 32 \\
$\mathrm{~V}_{34}$ & 28 & 35 & 22 & 14 & 6 & 26 \\
$\mathrm{~V}_{35}$ & 11 & 16 & 15 & 3 & 1 & 31 \\
$\mathrm{~V}_{36}$ & 25 & 31 & 20 & 7 & 5 & 35 \\
$\mathrm{~V}_{37}$ & 24 & 29 & 17 & 6 & 3 & 34 \\
$\mathrm{~V}_{38}$ & 10 & 12 & 7 & 17 & 14 & 20 \\
$\mathrm{~V}_{39}$ & 26 & 30 & 29 & 9 & 2 & 28 \\
& 22 & 25 & 23 & 11 & 12 & 39 \\
\hline & & & & & &
\end{tabular}

variant that may prove to be a significant addition in DE literature. Another possible direction of future work is to develop more powerful parent selection schemes that may improve the performance of these variants and to develop a mathematical model for DE variants that can serve as a standard.

\section{REFERENCES}

1. Storn R, Price K (1995) Differential Evolution: a Simple and Efficient Adaptive Scheme for Global Optimization over Continuous Spaces, ICSI, Berkeley, CA.
2. Brest J, Greiner S, Boskovic B, Mernik M, Zumer V (2006) Self-adapting control parameters in differential evolution: a comparative study on numerical benchmark problems. IEEE Trans Evol Comput 10, 646-57.

3. Price KV, Storn RM, Lampinen JA (2005) Differential Evolution: a Practical Approach to Global Optimization, Springer-Verlag, Berlin, Heidelberg.

4. Xia S, Zhou M, Li G (2011) A binary adaptive differential evolution algorithm for dynamic economic dispatch considering significant wind power. In: PowerTech, 2011 IEEE Trondheim, IEEE, pp 1-8.

5. Yang S, Qing A (2005) Design of high-power millimeter-wave TM/sub 01/-TE/sub 11/mode converters by the differential evolution algorithm. IEEE Trans Plasma Sci 33, 1372-6.

6. Zhao SZ, Qu BY, Suganthan PN, Iruthayarajan MW, Baskar S (2010) Multi-objective robust PID controller tuning using multi-objective differential evolution. In: 11th International Conference on Control Automation Robotics \& Vision, IEEE, pp 2398-403.

7. Chiou JP, Wang FS (1998) A hybrid method of differential evolution with application to optimal control problems of a bioprocess system. In: The 1998 IEEE International Conference on Evolutionary Computation Proceedings, IEEE, pp 627-32.

8. Fang Q, Chen D, Yu H, Wu X (2004) Differential evolution algorithm based on eugenic strategy and its application to chemical engineering. $J$ Chem Ind Eng 55, 598-602. [in Chinese]

9. Das S, Abraham A, Konar A (2008) Automatic clustering using an improved differential evolution algorithm. IEEE Trans Syst Man Cybern A 38, 218-37.

10. Du JX, Huang DS, Wang XF, Gu X (2007) Shape recognition based on neural networks trained by differential evolution algorithm. Neurocomputing 70, 896-903.

11. Karaboga N (2005) Digital IIR filter design using differential evolution algorithm. EURASIP J Adv Signal Process 2005, 856824.

12. Das S, Abraham A, Konar A (2008) Particle swarm optimization and differential evolution algorithms: technical analysis, applications and hybridization perspectives. In: Liu Y, Sun A, Loh HT, Lu WF, Lim EP (eds) Advances of Computational Intelligence in Industrial Systems, Springer, pp 1-38.

13. Zaharie D (2003) Control of population diversity and adaptation in differential evolution algorithms. In: Matousek R, Osmera P (eds) Proceedings of Mendel 9th International Conference on Soft Computing, pp 41-6.

14. Qin AK, Huang VL, Suganthan PN (2009) Differential evolution algorithm with strategy adaptation for global numerical optimization. IEEE Trans Evol Comput 13, 398-417.

15. Zhang J, Sanderson AC (2009) JADE: adaptive differential evolution with optional external archive. IEEE 
Trans Evol Comput 13, 945-58.

16. Das S, Abraham A, Chakraborty UK, Konar A (2009) Differential evolution using a neighborhood-based mutation operator. IEEE Trans Evol Comput 13, 526-53.

17. Wang Y, Cai Z, Zhang Q (2011) Differential evolution with composite trial vector generation strategies and control parameters. IEEE Trans Evol Comput 15, 55-66.

18. Mallipeddi R, Suganthan PN, Pan QK, Tasgetiren MF (2011) Differential evolution algorithm with ensemble of parameters and mutation strategies. Appl Soft Comput 11, 1679-96.

19. Islam SM, Das S, Ghosh S, Roy S, Suganthan PN (2012) An adaptive differential evolution algorithm with novel mutation and crossover strategies for global numerical optimization. IEEE Trans Syst Man Cybern B 42, 482-500.

20. Liu J, Lampinen J (2005) A fuzzy adaptive differential evolution algorithm. Soft Comput 9, 448-62.

21. Gong W, Cai Z, Ling CX, Li C (2011) Enhanced differential evolution with adaptive strategies for numerical optimization. IEEE Trans Syst Man Cybern B 41, 397-413.

22. Basu M (2008) Optimal power flow with FACTS devices using differential evolution. Int J Electr Power Energ Syst 30, 150-6.

23. Rocca P, Oliveri G, Massa A (2011) Differential evolution as applied to electromagnetics. IEEE Antenn Propag Mag 53, 38-49.

24. Qin H, Zhou J, Lu Y, Li Y, Zhang Y (2010) Multiobjective cultured differential evolution for generating optimal trade-offs in reservoir flood control operation. Water Resour Manag 24, 2611-32.

25. Suresh K, Kundu D, Ghosh S, Das S, Abraham A, Han SY (2009) Multi-objective differential evolution for automatic clustering with application to micro-array data analysis. Sensors 9, 3981-4004.

26. Selbaş R, Kızılkan O, Reppich M (2006) A new design approach for shell-and-tube heat exchangers using genetic algorithms from economic point of view. Chem Eng Process 45, 268-75.

27. Aslantas V, Kurban R (2010) Fusion of multi-focus images using differential evolution algorithm. Expert Syst Appl 37, 8861-70.

28. Ilonen J, Kamarainen JK, Lampinen J (2003) Differential evolution training algorithmfor feed-forward neural networks. Neural Process Lett 17, 93-105.

29. Vasundhara Mandal D, Ghoshal SP, Kar R (2013) Digital FIR filter design using hybrid random particle swarm optimization with differential evolution. Int $J$ Comput Intell Syst 6, 911-27.

30. Storn R, Price KV (1996) Minimizing the real functions of the ICEC'96 contest by differential evolution. In: Proceedings of IEEE International Conference on Evolutionary Computation, pp 842-4.

31. Storn R, Price K (1997) Differential evolution-a simple and efficient heuristic for global optimization over continuous spaces. J Global Optim 11, 341-59.

32. Ting CK, Huang CH (2009) Varying number of difference vectors in differential evolution. In: IEEE Congress on Evolutionary Computation, IEEE, pp 1351-8.

33. Sai Hanuman A, Hyd G (2010) Data clustering using almost parameter free differential evolution technique. Int J Comput Appl 8, 1-7.

34. Storn R (1996) On the usage of differential evolution for function optimization. In: Biennial Conference of the North American Fuzzy Information Processing Society, IEEE, pp 519-23.

35. Brest J, Bošković B, Greiner S, Žumer V, Maučec MS (2007) Performance comparison of self-adaptive and adaptive differential evolution algorithms. Soft Comput 11, 617-29.

36. Xin B, Chen J, Zhang J, Fang H, Peng ZH (2012) Hybridizing differential evolution and particle swarm optimization to design powerful optimizers: a review and taxonomy. IEEE Trans Syst Man Cybern C 42, 744-67.

37. Price KV (1996) Differential evolution: a fast and simple numerical optimizer. In: Biennial Conference of the North American Fuzzy Information Processing Society, IEEE, pp 524-7.

38. Zielinski K, Joost M, Laur R, Orlik B (2008) Choosing suitable variants of differential evolution and particle swarm optimization for the optimization of a PI cascade control. In: 11th International Conference on Optimization of Electrical and Electronic Equipment, IEEE, pp 55-62.

39. Gong W, Cai Z (2012) Adaptive parameter selection for strategy adaptation in differential evolution for continuous optimization. J Comput 7, 672-9.

40. Jeyakumar G, Shunmuga Velayutham C (2010) An empirical performance analysis of differential evolution variants on unconstrained global optimization problems. Int J Comput Inform Syst Ind Manag Appl 2, 77-86.

41. García-Martínez C, Rodríguez FJ, Lozano M (2011) Role differentiation and malleable mating for differential evolution: an analysis on large-scale optimisation. Soft Comput 15, 2109-26.

42. Gong W, Cai Z, Jia L, Li H (2011) A generalized hybrid generation scheme of differential evolution for global numerical optimization. Int J Comput Intell Appl 10, 35-65.

43. Podoba T, Tomsu L, Vlcek K, Heczko M (2010) Surface reconstruction by means of AI. In: International Conference on Computational \& Experimental Engineering and Sciences, vol 15, 111-22.

44. Zelinka I, Senkerik R, Bialic-Davendra M, Davendra D (2010) Chaos driven evolutionary algorithm for the traveling salesman problem. In: Davendra D (ed) Traveling Salesman Problem, Theory and Applications, InTech, pp 55-70. 
45. Triguero I, García S, Herrera F (2011) Differential evolution for optimizing the positioning of prototypes in nearest neighbor classification. Pattern Recogn 44, 901-16.

46. Zhao SZ, Suganthan PN, Das S (2011) Self-adaptive differential evolution with multi-trajectory search for large-scale optimization. Soft Comput 15, 2175-85.

47. Almeida-Luz S, Vega-Rodríguez MA, Gómez-Pulido JA, Sánchez-Pérez JM (2008) Applying differential evolution to the reporting cells problem. In: Proceedings of the International Multiconference on Computer Science and Information Technology, IEEE, pp 65-71.

48. Mendes R, Mohais AS (2005) DynDE: a differential evolution for dynamic optimization problems. In: The 2005 IEEE Congress on Evolutionary Computation, IEEE, pp 2808-15.

49. Ao YY, Chi HQ (2010) An adaptive differential evolution algorithm to solve constrained optimization problems in engineering design. Engineering 2, 65-77.

50. Mandal A, Das S, Abraham A (2011) A differential evolution based memetic algorithm for workload optimization in power generation plants. In: 11th International Conference on Hybrid Intelligent Systems, IEEE, pp 271-6.

51. Epitropakis MG, Tasoulis DK, Pavlidis NG, Plagianakos VP, Vrahatis MN (2011) Enhancing differential evolution utilizing proximity-based mutation operators. IEEE Trans Evol Comput 15, 99-119.

52. de Oliveira GTS, Saramago SFP (2007) A contribution to the study about differential evolution. Ciênc Eng 16, 1-8.

53. Weber M (2010) Parallel global optimization: structuring populations in differential evolution. $\mathrm{PhD}$ thesis, Univ of Jyvaskyla, Finland.

54. Triguero I, García S, Herrera F (2010) A preliminary study on the use of differential evolution for adjusting the position of examples in nearest neighbor classification. In: IEEE Congress on Evolutionary Computation, IEEE, pp 1-8.

55. Goudos SK, Zaharis ZD, Yioultsis TV (2010) Application of a differential evolution algorithm with strategy adaptation to the design of multi-band microwave filters for wireless communications. Progr Electromagn Res 109, 123-37.

56. Fialho A, Schoenauer M, Sebag M (2010) FitnessAUC bandit adaptive strategy selection vs the probability matching one within differential evolution: an empirical comparison on the bbob-2010 noiseless testbed. In: Proceedings of the 12th Annual Conference Companion on Genetic and Evolutionary Computation, Oregon, USA, pp 1535-42.

57. Fialho A, Gong W, Cai Z (2010) Probability matchingbased adaptive strategy selection vs uniform strategy selection within differential evolution: an empirical comparison on the bbob-2010 noiseless testbed. In: Proceedings of the 12th Annual Conference Companion on Genetic and Evolutionary Computation, Oregon, USA, pp 1527-34.

58. Elsayed SM, Sarker RA, Essam DL (2011) Differential evolution with multiple strategies for solving CEC2011 real-world numerical optimization problems. In: 2011 IEEE Congress on Evolutionary Computation, IEEE, pp 1041-8.

59. Elsayed SM, Sarker RA, Essam DL (2011) Multioperator based evolutionary algorithms for solving constrained optimization problems. Comput Oper Res 38, 1877-96. 